\title{
Ratings, Reviews, and the Marketing of New Products
}

\author{
Itay P. Fainmesser ${ }^{1} \quad$ Dominique Olié Lauga ${ }^{2} \quad$ Elie Ofek ${ }^{3,4}$
}

September 8, 2020

${ }^{1}$ Johns Hopkins Carey Business School and The Economics Department, Johns Hopkins University, Baltimore, MD 21202. E-mail: Itay_Fainmesser@jhu.edu

${ }^{2}$ Cambridge Judge Business School, University of Cambridge, Cambridge CB2 1AG, UK. Email: d.lauga@jbs.cam.ac.uk.

${ }^{3}$ Harvard Business School, Soldiers Field, Boston, MA 02163. E-mail: eofek@hbs.edu

${ }^{4}$ The authors thank participants at the SICS conference (UC Berkeley), Voya Financial Colloquium (University of Connecticut), Zero Decade Marketing Theory Symposium, 2019 Invitational Pricing Symposium, and University Carlos III de Madrid marketing seminar for valuable suggestions. The authors also thank David Godes for helpful comments. 


\title{
Ratings, Reviews, and the Marketing of New Products
}

\begin{abstract}
We study how user-generated content (UGC) about new products impacts a firm's advertising and pricing decisions as well as the effect on profits and market dynamics. We construct a twoperiod model, where consumers value quality and are heterogeneous in their taste for the new product's positioning, and examine three information transfer structures across generations: no information (Benchmark); average rating (AR); and the joint distribution of ratings and taste locations (Reviews). First, we show that in the AR case the firm advertises to a smaller set of consumers and prices higher relative to the other cases. This occurs because the firm has an incentive to use advertising strategically to bump up the first-generation average rating in order to increase second-generation quality perceptions. The firm charges a higher first-period price as consumers who are relatively close to the product's location are advertised to. Second, we find that as more information is transferred across generations there is a greater likelihood that average ratings will exhibit an increasing pattern over time. This happens because with Reviews second-generation consumers are able to base purchase decisions on the product's positioning in addition to its quality. Interestingly, due to the firm's narrower advertising strategy in the AR case and the richer UGC in the Reviews case, average ratings will exhibit a reversal over time: higher in the AR case in the first period but higher in the Reviews case in the second period. Third, a firm's expected profits can exhibit a non-monotonic relationship with respect to the amount of UGC transferred: highest in the AR case if the marginal return on advertising is high (e.g., when advertising is cheap to execute, consumers are insensitive to product fit, or consumers greatly value quality), but highest in the Reviews case when the return on advertising is low. We then examine a setup whereby second-generation consumers become aware of the product through social interaction with first-generation buyers. We find that advertising and social contagion interact - the firm generally has a greater incentive to advertise as homophily increases, i.e., as social interactions occur between consumers who more likely share similar preferences. However, this pattern can reverse with Reviews. The paper discusses several robustness checks and model extensions, and concludes by highlighting the managerial implications of the results.
\end{abstract}

Keywords: Online Reviews, Product Ratings, Social Networks, Word-of-Mouth, Advertising, Quality, Pricing, User-generated Content 


\section{Introduction}

One of the hallmarks of the digital transformation witnessed over the past two decades is the fact that people are more easily and immediately connected to each other than ever before. While this has resulted in the rise of several social media platforms over which individuals share various personal experiences with friends, family members and colleagues, it has also had profound implications for businesses. In particular, consumers can use online tools to share impressions of the products and services they purchased, often on the vendor's website or on ecommerce platforms, and prospective consumers can examine these impressions and incorporate them into their own decision making. Indeed, recent data suggests that $79 \%$ of U.S. internet users check customer ratings and up to $55 \%$ check detailed reviews to inform their purchase decisions. ${ }^{1}$ Consumer generated content can thus impact demand and hence firm fortunes.

For instance, if a recently launched new product receives an average rating of 4.5 (out of 5) based on the input from early buyers, we might expect later consumers to be more inclined to buy it compared to a product in the same category that receives an average rating of 3.8. That said, if presented with the option, buyers might also take the time to write detailed reviews about the new product and why they liked (or disliked) it given their particular preferences. If subsequent consumers read these reviews, they could arrive at a different conclusion regarding the product than had they relied solely on the average rating. Specifically, though some aspects of the product are appreciated by all consumers and can be summarized by a 'quality' level (e.g., the speed/performance of a computer, the battery life of a smartphone, the amount of glitches in a new mobile game, or the effectiveness of safety features in a car), other product aspects may fit the tastes of some consumers but not those of others (e.g., the exterior design of a computer, the user interface of an electronic device, the characters and visuals of a mobile game, or the plot line of a movie). As a result, several buyers might provide a low rating for a product because it does not fit their particular tastes, despite its relatively high quality. A later arriving consumer, who only observes the average rating without more detailed information on what drove it, might be worried that the product is of poor quality and be deterred from buying it. But if this consumer reads the full reviews and figures out that the reason for the mediocre average rating had to do with a product taste/fit issue that does not apply to them, they may in fact decide to make a purchase. Indeed, there is evidence, e.g., in the movie category,

\footnotetext{
${ }^{1}$ Source: Better Business Bureau and Nielsen, "BBB Trust Sentiment Index," October 2, 2017; and Performics and Northwestern University's Intent Lab, "Digital Satisfaction Index," August 4, 2017.
} 
that average ratings impact the market performance of new products and that reviews often contain relevant information which can further affect consumer behavior (see, e.g., Chintagunta et al., 2010; Moon et al., 2014; Rocklage and Fazio, 2020). It is further intriguing to note that, in reality, we find that some companies (such as Best-Buy or Chewy) require consumers who want to provide online input to write comments in addition to indicating a rating, while other companies (such as Wal-Mart or Target) allow forgoing comments and providing ratings only. From the firm's perspective, the question is whether the nature of the information transferred across consumer generations should affect any of its strategic actions.

In this paper, we examine the interplay between the type of new product assessments communicated by buyers and a firm's strategic actions, namely advertising and pricing. We focus on the following research questions.

- Does a firm have a weaker or stronger incentive to expend on advertising when consumers have access to reviews vs. just average ratings? Does this incentive depend on the nature of social interaction across generations?

- Does the information transferred across generations affect how aggressively a firm prices a new product and how its pricing evolves over time?

- What should we expect in terms of average rating dynamics? Specifically, under what conditions will the pattern of average ratings increase vs. decrease across periods?

- How does the type of information transferred across generations impact firm profits and consumer welfare? Should we expect lower profits and greater consumer surplus when more information is transferred?

To address these questions, we construct a two-period model where a firm introduces a new product in the first period and needs to decide how extensively to advertise it to firstgeneration consumers and how much to charge for it, with the ability to change its pricing over time. The product's positioning (along a circle) is ex-ante known to the firm whereas its quality is uncertain prior to market consumption. Consumers are homogeneous in their valuation for quality, yet heterogeneous in their tastes for the product's positioning and in their base valuation for consuming in the category.

We examine three possible information transfer structures between first-generation consumers who purchased the product (i.e., buyers) and second-generation consumers: a Bench- 
mark setting where no buyer evaluations are passed on; an Average Rating (AR) setting where only the average net consumption utility of buyers is passed on; and a Reviews setting where the joint distribution of net utilities (ratings) and taste locations of buyers is passed on (e.g., via posted comments/pictures/videos). We first solve a basic model where all second-generation consumers are aware of the product and later examine a setting where second-generation awareness only occurs through social interaction with previous buyers.

Our analysis of the basic model yields several interesting findings. First, we show that demand in period 2 is the same in size regardless of whether an average rating or reviews are transferred; yet the set of buyers is different. In the AR case, second-generation buyers are uniformly spread around the circle, whereas in the Reviews case they are concentrated around the consumer location that best matches the product's positioning. Second, we show that in the AR case a firm targets a smaller set of consumers with advertising in period 1 compared to the Benchmark and Reviews cases. This occurs because in the AR case second-generation consumers infer quality based on the average rating they observe and their belief about the firm's advertising level. This gives the firm an incentive to use advertising strategically to increase the period 1 average rating in an effort to boost period 2 demand; resulting in a smaller advertising interval selected in equilibrium. Furthermore, in the AR case the firm is induced to price higher in period 1 because it expects to sell to consumers closer to the product's position and who therefore have higher valuations.

Third, we find that as more information is transferred, there is a greater likelihood that average ratings will exhibit an increasing pattern over time. This happens because with more information, i.e., with Reviews rather than just AR as input, second-generation consumers are able to make decisions based on product positioning as well as quality. This results in second-generation purchases, and subsequent ratings, by consumers with higher valuations on average. Furthermore, due to the narrower advertising strategy in the AR case, but richer user generated content (UGC) consumers have at their disposal in the Reviews case, average ratings will exhibit a reversal over time: they will be highest in the AR case in period 1 but highest in the Reviews case in period 2. That said, even with Reviews average ratings can decrease across periods and, surprisingly, such a declining pattern happens when the product is of relatively high quality. As for period 2 pricing, while in the Benchmark case prices always decline to accommodate the greater consumer awareness, in the AR and Reviews cases they may increase or decrease depending on the quality revealed. 
Finally, we show that expected profits can exhibit a non-monotonic relationship with respect to the amount of UGC information available. Specifically, if the marginal return on advertising spending is high - which occurs if the cost to advertise is modest, consumers are insensitive to product fit, or consumers greatly value quality - expected profits increase between the Benchmark and AR settings and then decrease with Reviews, i.e., highest in the AR case. Yet if the marginal return on advertising spending is low, expected profits are highest in the Reviews setting. The robustness of these findings to relaxing key model assumptions is discussed.

We then explore an alternative setting in which second-period awareness is contingent upon connecting with a first-period buyer. Specifically, each second-generation consumer interacts with a sample of first-generation consumers whose location along the circle is either identical to her own, reflecting homophily, or randomly drawn, and becomes aware if at least one of them purchased the product. In this setting, we find that the firm has a greater incentive to advertise in period 1 than in the basic model and, interestingly, casts the widest advertising net when no information is transferred across generations (Benchmark case). Furthermore, the degree of homophily plays a critical role: the more second-generation consumers expect to be paired with first-generation consumers who are like them in terms of preference location, the more broadly the firm tends to advertise. This is true in the Benchmark and AR cases because aware second-generation consumers do not know the product's exact position but presume they are close to it when homophily is strong. The firm seeks to exploit this fact by advertising to more first-generation consumers who can serve as social contagion agents. However, the reverse pattern can occur in the Reviews case because aware consumers are able to learn the exact product position from UGC and, if located far away from it, are unlikely to buy; thus prompting the firm to target its advertising more narrowly. Lastly, we show that with social interactions average ratings are more likely to exhibit an increasing pattern over time than in the basic model and that expected profits are highest in the AR case.

The rest of the paper is organized as follows. In the next section, we relate our work to pertinent literature. We then describe the basic setup and present the main findings from analyzing this model. We close this section by outlining several robustness checks and extensions. Subsequently, we examine a setting where awareness in period 2 is a function of social interactions across generations. We end with concluding remarks and managerial implications. All proofs are relegated to the Appendix or Technical Appendix. 


\section{Literature Review}

Our work contributes to the growing body of literature on the strategic implications of online customer-generated evaluations of products and services, particularly research on how such evaluations affect a firm's marketing mix variables. Sun (2012) studies how the average and variance of ratings impact firm pricing and profits. In her model, all consumers are aware of a new product and its location, but are uncertain about its quality and about the common mismatch cost parameter along the horizontal dimension. She finds that a higher average rating results in higher prices and profits (consistent with the findings of Chevalier and Mayzlin, 2006). Yet greater variance also yields higher profits when the average rating is low enough. Our work differs from Sun (2012) in that we do not assume that consumers ex-ante know the product exists and hence the firm has to engage in costly advertising. Moreover, consumers in our model know the mismatch cost parameter but are ex-ante uncertain about the product's position. We then examine how the nature of buyer evaluations (average ratings vs. full reviews) interacts with advertising and pricing, as well as the role of WOM across generations.

Kuksov and Xie (2010) examine a setting where consumers in period 2 infer the uncertain portion of a product's value (quality) from the observed average rating of period 1 buyers. All consumers are assumed to be aware of the product, and the firm can try to affect ratings by offering post-purchase extras (called "frills") and by lowering price. They find that frills are only offered to period 1 buyers if the market exhibits sufficient growth, i.e., there are more consumers in period 2 than in period 1; otherwise the firm will lower price (as compared to a single-period firm). The intuition being that because frills occur post-purchase and are incurred for all period 1 buyers, offering them makes sense only if the effect this can have on the average rating will impact many more consumers in period 2. In our model, consumers are uncertain about quality as well as product fit. Unlike frills, which directly impact the buyers' net utility, in our setup a key variable under the firm's control is advertising, which determines who will become aware of the product (and aware consumers must form beliefs about the firm's actions). This, in turn, affects who may buy and provide UGC. Importantly, our focus is on comparing different UGC structures: no information (Benchmark), partial information (AR) and full information (Reviews). We find that, compared to the other information cases, the firm in the AR case restricts advertising even when the market size stays flat or shrinks across periods (see Section 
3.2 ) and it also has a tendency to charge higher prices in period $1 .^{2}$ In some sense, the firm in our model is able to endogenize the market size of potential period 1 buyers. Moreover, the actions taken in the AR case, intended to influence ratings, can either backfire or avail the firm compared to the Reviews case; thus a non-monotonic relationship between the amount of UGC conveyed and profits is possible. We further explore average rating dynamics and characterize a reversal: under AR the average rating is higher in period 1 but lower in period 2 compared to the Reviews case. This result is directly linked to our modeling of horizontal uncertainty in addition to vertical (quality) uncertainty. These profit and rating dynamic issues are not part of the analysis in Kuksov and Xie (2010). Lastly, by micro-modeling social interactions across generations, we are able to examine how consumer expectations about who they interact with affect the firm's incentives to advertise. In more recent work, Kuksov and Liao (2019) examine how consumer inferences from opinion leader (expert) recommendations affect optimal product variety. An expert bases his/her recommendation on product quality (which is ex-ante uncertain) as well as fit to idiosyncratic tastes (which is privately known). While, in general, greater variety increases the chances of a positive recommendation, this may depress consumers' inference on quality. The authors show that it may therefore be optimal for the firm to offer a finite number of product variants. In our model, second-generation consumers in the AR case are able to infer product quality from the average rating but remain uncertain about the product's location, and hence the fit to their tastes. This results in lower period 2 ratings compared to the Reviews case, yet may be more profitable. Thus, although in both these past works the firm also takes actions that affect the inferences of second-generation consumers (from UGC or expert recommendations), our setup - with a focus on comparing the type of information transferred - and decision variables are quite different, leading to substantively distinct findings.

Chen and Xie (2008) examine a seller's incentive to provide full or partial information regarding the attributes of its product in the presence of consumer reviews. Their model assumes full awareness and differentiates between expert consumers, who can process the firm's advertising, and novices, who only use reviews from period 1 consumers to determine product match. They show that the firm's optimal strategy depends on marginal product costs, the accuracy of consumer reviews, and segment sizes. In our model, second-generation consumers

\footnotetext{
${ }^{2}$ Charging higher prices in the AR case holds strictly in our model when price history is observable. If price history is unobserved, the firm in the AR case also has an incentive to reduce price to affect consumer inferences, thus it might select a lower price than in the other cases (see Section 3.2).
} 
can only glean information about the product from UGC, while production costs do not play a role. Joshi and Musalem (2020) construct a model in which the firm knows its quality at the outset and advertising serves a signaling role, while pricing is exogenous. They find that in the presence of WOM a high-quality firm may engage in more aggressive advertising in order to signal its type. In our model, by contrast, advertising plays an awareness-generating role and its extent is unobserved by consumers (hence it cannot serve as a signal), and we vary the amount of information transferred from first-generation buyers. We find that partial information results in less aggressive firm behavior (smaller advertising interval and higher prices). In an extension, we examine how the nature of social interactions matters for advertising intensity.

Our work is also related to Godes (2017) who looks at how WOM affects a firm's product quality decision. He finds that the optimal quality chosen depends on whether WOM disproportionately impacts lower- or higher-valuation consumers and on whether it serves to expand awareness or inform about quality. ${ }^{3}$ Jiang and Yang (2019) examine a setting where period 2 consumers learn the product's quality from period 1 buyers and the firm has private information on its marginal cost of quality provision (which it can signal through price). Their analysis reveals that it may be optimal for a highly efficient firm (low marginal cost) to reduce quality when its efficiency is known compared to when it is not. In our model, quality is exogenous (though uncertain) and the focus is on how the type of information transferred between consumers affects a firm's targeted advertising and pricing decisions. Our model further includes a horizontal fit dimension that is absent in Godes (2017) and Jiang and Yang (2019).

Lastly, our work is related to research examining patterns of rating dynamics. As expressed in Godes and Silva (2012), and references therein, some researchers have found that average ratings tend to decrease over time while others have found the opposite. Godes and Silva (2012) empirically tease out temporal and sequential factors that may affect average ratings. Our theoretical exploration is sequential in nature, and reveals that the pattern of average ratings critically depends on how much information is conveyed in UGC as well as on realized quality; noting that higher quality products tend to generate a decreasing pattern.

To summarize, our work contributes to the literature on user-generated evaluations along four key dimensions. First, we examine different UGC information structures in a manner that has not been looked at before at our level of detail in a single context, namely an average rating vs. full reviews. Second, we explore the interaction between customer-generated evaluations and

\footnotetext{
${ }^{3}$ Godes (2017) treats advertising separately from WOM, while we examine the interaction between them.
} 
the firm's advertising and pricing decisions. Third, we shed new light on the evolution of average ratings and delineate conditions for various patterns to emerge. Fourth, we combine product evaluations from previous buyers with the process of social interaction across generations.

\section{Basic Model}

There are two generations of consumers, who live in periods $t=1,2$ respectively. We consider a Salop model such that each generation reflects a new unit mass of consumers, who are spread uniformly on a circle of length 4 . We discuss the possibility of some consumers carrying over from period 1 to period 2 and generations of unequal size in Section 3.2.

The firm has developed a new product that is positioned at location $y$, which is uniformly drawn from $[0,4]$. The location of the new product is unobserved by consumers but known to the firm. The product is of quality $q$, drawn from a uniform distribution over [0,2] (independently of the draw of $y$ ). The quality $q$ is initially unobservable to the firm, and becomes known to buyers, i.e., consumers who purchase and try the product, thus reflecting an experience good. We assume for simplicity that the firm produces this product at zero marginal cost.

The setting we have in mind is one where the internal new product planning process defines the intended positioning (see, e.g., Ulrich and Eppinger, 2015; Urban and Hauser, 1993), which is why the firm knows the consumer location the product best matches. However, the firm is unsure prior to actual market consumption how "good" the new product will turn out to be along the quality (vertical) dimension. ${ }^{4}$ For instance, a firm may produce a movie with a plot and comic elements of a particular style; hence it determines the "taste" for which the product is best suited for. The firm is uncertain, however, prior to the movie's release and customers experiencing it, just how favorably it will be deemed with respect to various quality measures (acting, wardrobe, directing, special effects, etc.). In other words, given the specific sub-genre (which the firm knows), whether the movie will be regarded a "hit" or a "dud" is ex-ante uncertain. Examples include the 2017 film 'Wonder Woman' that greatly exceeded expectations vs. the 2011 film 'Green Lantern' that disappointed (Hughes, 2017; Barnes, 2011). Similar examples come to mind in other markets where new product activity

\footnotetext{
${ }^{4}$ It is easy to extend the model to allow the product's expected quality to be related to some measure of ex-ante R\&D effort (for example, the product's quality will fall within a range of possible values around a mean level that is a function of $R \& D$ ). But as long as the firm remains uncertain about the exact outcome of this $\mathrm{R} \& \mathrm{D}$, in terms of the realized appreciation for the product by consumers, our results will carry through.
} 
is prevalent, e.g., consumer electronics and cars. When Samsung launched its Galaxy Note 7, the added capabilities and features delivered benefits aimed mainly at people with a particular set of preferences. However, as more batteries malfunctioned and put users at risk, quality perceptions of the new product changed drastically (Wiggers, 2017). Similarly, the Microsoft Surface Book 2 was designed to best serve consumers with certain use cases. Yet a hardware bug, which led to battery draining in some situations, was revealed post launch (Warren, 2017). The Ford Escape, on the other hand, an SUV positioned to fit the tastes of small families with particular driving habits, surprised consumers for the better with respect to quality after its release (CarGurus, 2006). In Section 3.2, we discuss several ways to relax the assumption that the firm knows the product's location ex-ante while consumers don't.

To formalize the above characterization, let the utility of consumer $i$ at location $x$ be zero if she does not purchase the good, and if she purchases the good her utility is

$$
u_{x}=\alpha_{i}+\beta q-d(x, y) \tau-p
$$

where $d(x, y) \equiv \min \{|x-y|, 4-|x-y|\}$ is the shortest distance between the consumer's location $x$ and the product's position $y, \tau$ is the marginal disutility from consuming a product that is different from the ideal point (a.k.a., the mismatch cost parameter), $\beta$ is the marginal valuation for quality, $\alpha_{i} \sim U[0,1]$ is an idiosyncratic parameter known only to consumer $i$ that reflects her base valuation or attraction for products in the category, and $p$ is the price of the good. A consumer has no utility from purchasing more than one unit (and the good is in a non-repeat purchase category).

At the outset of the game, consumers are unaware of the new product's existence. The firm has the ability to engage in costly targeted advertising to reach a subset of them and make them aware. The timeline of the game is as follows: In period $t=1$, the firm chooses a price $p_{1}$ and an advertising strategy consisting of a targeted interval of length $4 z$ (where $z \in[0,1]$ ) centered around the product's position $y .{ }^{5}$ First-generation consumers who fall within the advertising interval learn about the existence of the product and its price $\left(p_{1}\right)$, yet do not observe the firm's targeting strategy $(z)$ and must form a belief about it (denoted $z^{e}$ ) to calculate their expected

\footnotetext{
${ }^{5}$ The ability to target advertising communications based on consumer characteristics and preferences is becoming increasingly more precise and common on digital platforms, such as Facebook and Google. The assumption that the advertising interval is centered around $y$ is without loss of generality. If we allow the firm to choose the center of the interval as well as its length we find that it will always choose the product's position $y$. The proof (available from the authors upon request) follows a simple revealed preference argument.
} 
utility from purchasing the product. The advertising cost, $A(z)$, is increasing and sufficiently convex in the size of the targeted group to guarantee that the solution is interior (in particular, we assume $A(0)=0 ; A^{\prime}(z)>0 ; A^{\prime \prime}(z)>\frac{\tau}{\beta}$; and $\left.A^{\prime \prime}(1)>0.4\right)$. Once advertising takes place, aware consumers make simultaneous purchasing decisions. Buyers experience the product, and after realizing quality and fit know their net utility from consumption.

At the start of period 2, a fraction $\gamma$ of second-generation consumers become aware of the new product. This captures scenarios where through observation, word-of-mouth (WOM), or exposure to press coverage some second-generation consumers learn about the existence of the product. For ease of exposition, in this section we solve for the case of $\gamma=1$, i.e., all second-generation consumers become aware (the results are qualitatively unchanged for any $0<\gamma \leq 1$ ). In Section 4, we micro-model the WOM process, which leads to only a subset of second-generation consumers becoming aware of the product.

Let second-generation consumers observe a summary statistic of information obtained from first-generation buyers. We consider three options:

1. Benchmark: second-generation consumers only learn about the product's existence.

2. Average Rating (AR): second-generation consumers learn about the product's existence and observe the mean net utility of first-generation buyers.

3. Reviews: second-generation consumers learn about the product's existence and observe the joint distribution of the utilities and locations of first-generation buyers.

To elaborate, we assume that, given the visible consumption of the product by at least some first-generation consumers, the baseline information transferred across generations is that the product exists (Benchmark case). When first-generation buyers also indicated a summary of their overall assessment of the product linked to their net consumption utility, for example through an online rating system, we assume that second-generation consumers have access to the mean of these utilities through an average rating score (AR case). ${ }^{6}$ Lastly, if first-generation buyers not only provided a measure of their net utility by rating the new product but also wrote reviews in which they discussed its fit with their preference location, second-generation consumers upon reading these comments can deduce the true quality and position of the product

\footnotetext{
${ }^{6}$ In many online systems, consumers choose from and/or observe a discrete set of rating options. Our results extend to such environments - see Section 3.2 and the Technical Appendix for details.
} 
(Reviews case). ${ }^{7}$ The firm has access to the UGC from past buyers, and can set a new price $\left(p_{2}\right)$ at the start of period 2. Prices are assumed to be observable, hence second-generation consumers know $\left(p_{1}, p_{2}\right)$ and make simultaneous purchase decisions. In Section 3.2 we discuss other pricing structures, including a non-observable price history.

In making pricing and advertising decisions, the firm seeks to maximize total expected profits across periods with no discounting. To ensure interior solutions, we assume that $\tau$, the location mismatch parameter, and $\beta$, the marginal sensitivity to quality, are smaller than $1 / 5$. These upper bounds imply that: 1 . even in the farthest location from the product's position there is always some consumer who, if aware of the product, buys it, and 2. even in the closest location to the product's position there is some consumer who does not buy it. In other words, the product is not ex-ante restricted to a niche population along the circle nor is it appealing to everyone at the equilibrium price (see the Technical Appendix for formal details).

\subsection{Analysis}

\subsubsection{Demand}

Given the prices and advertising level the firm chooses, we can derive closed-form solutions for the demand in each period. In particular, period 1 demand can be written $\mathrm{as}^{8}$

$$
D_{1}\left(z, p_{1}\right)=z\left(1+\beta-z^{e} \tau-p_{1}\right)
$$

where $z^{e}$ reflects consumers' expectation of the advertising interval chosen by the firm. Firstgeneration consumers targeted with advertising use this value (as they do not observe $z$ directly) to assess their distance to the product's location (in equilibrium $z^{e}$ will correspond to the true value $z$ chosen by the firm).

The mean net utility, i.e., average rating, of first-generation buyers is, in turn,

$$
\mu_{1}(z)=\frac{1+\beta(2 q-1)-\left(2 z-z^{e}\right) \tau-p_{1}}{2} .
$$

\footnotetext{
${ }^{7}$ We assume that second-generation consumers can't perfectly observe the total number of first generation consumers who were made aware of the product through advertising $(z)$. This is highly realistic, as even on platforms such as Amazon it is not possible to figure out from a product's sales rank nor from the number of customers who provided feedback exactly how many people were targeted with ads.

${ }^{8}$ See the Technical Appendix for the formal derivation of expressions (1) and (2). Note that we do not incorporate strategic behavior on the part of first-generation buyers meant to influence second-generation consumers. Consequently, period 1 demand is independent of the information transmitted across generations.
} 
An important observation is that $\frac{\partial \mu_{1}}{\partial z}=-\tau<0$, and therefore the average rating decreases in the size of the advertising interval the firm chooses. This is intuitive: a larger advertising interval (a greater $z$ ) increases the average distance between those consumers aware of the product and its position on the circle. This leads to a decrease in the average utility of buyers and is a function of consumers' sensitivity to product taste mismatch $(\tau)$. Further note that $\frac{\partial \mu_{1}}{\partial z^{e}}=\frac{\tau}{2}>0$, reflecting the fact that when targeted first-generation consumers believe the firm chooses a larger interval, they expect to be farther away from the product than they actually are. And the more consumers care about product fit, the more this will deter those with low idiosyncratic utility (with low $\alpha_{i}$ ) from purchasing; thus the average rating will rise. The dependence of $\mu_{1}$ on price is straightforward.

To analyze the behavior of second-generation consumers, note that the three informational cases differ in the type of inferences these subsequent consumers are able to make. In the Benchmark case, second-generation consumers only learn that the product exists and its price history, but are unaware of $q$ and $y$. In the AR case, given the average rating they observe, second-generation consumers can additionally establish an estimate for quality, $q^{e}$, based on what they expect the size of the ad interval to have been $\left(z^{e}\right)$; but not the location of this interval or who was targeted. ${ }^{9}$ Second-generation consumers interpret a higher average rating $\left(\mu_{1}\right)$ as, in part, reflecting greater quality. In particular, through the inverse function for $\mu_{1}\left(z^{e}\right)$ in (2) we have $q^{e}=\frac{p_{1}+\beta+2 \mu_{1}+z^{e} \tau-1}{2 \beta}$, which when substituting in $\mu_{1}(z)$ yields $^{10}$

$$
q^{e}=\frac{\beta q-\tau\left(z-z^{e}\right)}{\beta}
$$

Note from (3) that the quality inferred by second-generation consumers in the AR case is increasing in the true quality. This is because higher quality affects the net utility of firstgeneration buyers who experienced the product and learned $q$, resulting in a higher average rating. The dependence of $q^{e}$ on $z^{e}$ is as follows. For a given average rating observed, an increase in $z^{e}$ suggests that many first-generation buyers were farther away from the product's

\footnotetext{
${ }^{9}$ Digital advertising methods today allow for very tailored messages, yet who receives these messages is only known to the targeted consumer (i.e., unknown to second-generation consumers).

${ }^{10}$ We note that off-the-equilibrium-path $\frac{\beta q-\tau\left(z-z^{e}\right)}{\beta}$ might be negative or larger than 2, especially if the actual quality is close to 0 or 2 respectively. Such expectations for $q$ are not valid in the model. Since these are zero probability events, we assume throughout that consumers' off-path beliefs with respect to $q$ are captured by $q^{e}=\min \left\{\max \left\{0, \frac{\beta q-\tau\left(z-z^{e}\right)}{\beta}\right\}, 2\right\}$.
} 
position, which should negatively impact net utility. Therefore, quality must have been high in order to compensate for that.

Finally, in the Reviews case, second-generation consumers observe the joint distribution of first-generation buyers' utilities and locations, and are thus able to accurately infer $q$ and $y$.

We solve for the Perfect Bayesian Equilibria $(\mathrm{PBE})$ of the game, requiring that relevant consumer expectations on firm variables be fulfilled and that the firm not have an incentive to deviate from its proposed equilibrium strategy given consumers' beliefs. Hence, consumers must have correct expectations with respect to $z$ (and infer quality correctly, $q^{e}=q$, from UGC when relevant). We focus on pure strategy equilibria, and accordingly on deterministic consumer beliefs over firm actions. ${ }^{11}$

We now turn to analyzing period 2 demand under the three informational cases. We denote by $D_{2}^{0}, D_{2}^{A R}, D_{2}^{R}$ period 2 demand in the Benchmark, Average Rating, and Reviews cases, respectively. In the Technical Appendix, we establish that

$$
D_{2}^{0}\left(p_{2}\right)=1+\beta-\tau-p_{2}, \quad D_{2}^{A R}\left(p_{2}\right)=1+\beta q^{e}-\tau-p_{2}, \quad \text { and } \quad D_{2}^{R}\left(p_{2}\right)=1+\beta q-\tau-p_{2} .
$$

As can be gleaned from (4), the demand in period 2 differs across information structures. In particular, when neither the average rating nor reviews are transmitted (Benchmark case) the demand is independent of quality and consumers use the ex-ante expected value $(E(q)=1)$. However, when more information is transmitted, demand depends on either inferred quality $q^{e}$ (AR case) or on actual quality $q$ (Reviews case). In equilibrium, as expectations are fulfilled, $z^{e}=z$ and $q^{e}=q$, and period 2 demand in the AR case becomes

$$
D_{2}^{A R}\left(p_{2}\right)=1+\beta q-\tau-p_{2}
$$

From (4) and (5) it is apparent that, holding price constant, realized period 2 demand (in terms of its size) is the same in the AR and Reviews cases. However, it is important to stress that, even if prices are identical, the set of buyers is different. In the AR case, buyers are uniformly spread around the circle (as they do not know the position of the product and only its quality is inferred), whereas in the Reviews case buyers are centered around the product's

\footnotetext{
${ }^{11}$ We assume that if consumers were to observe a firm action (e.g., price) that is inconsistent with what their beliefs imply the firm should have selected (which is a zero probability event), they would maintain their original belief over $z$. See proof of Lemma TA-2 in the Technical Appendix for a discussion of how beliefs are treated in the PBE we solve for and its robustness.
} 
position. Note further that since $E(q)=1$, holding price constant, ex-ante expected demand in period 2 is equal across the three informational cases. However, realized (ex-post) period 2 demand will be lower in the Benchmark case when $q>1$, and the reverse is true when $q<1$.

We point out that in the AR and Reviews cases second-generation consumers establish correct deterministic posteriors over the product's quality, given the information they observe from first-generation buyers. As a result, the firm cannot affect consumers' quality perceptions by distorting $p_{2}$. In the Benchmark case, with no UGC, neither the firm nor consumers can infer quality, and the firm optimally selects $p_{2}$ in a way that does not depend on $q .{ }^{12}$

\subsubsection{Marketing Decisions: Advertising and Pricing}

In period 2 , the firm chooses the price $p_{2}$ to maximize its expected profits $p_{2} D_{2}\left(p_{2}\right)$, taking into account the product's quality if known. In the Benchmark case, this amounts to solving

$$
p_{2}^{0}=\underset{p_{2}}{\arg \max } p_{2} D_{2}^{0}\left(p_{2}\right)=\underset{p_{2}}{\arg \max } p_{2}\left(1+\beta-\tau-p_{2}\right) .
$$

A first-order analysis yields $p_{2}^{0}=\frac{1+\beta-\tau}{2}$.

At the beginning of period 1, the firm considers how demand plays out and chooses the price $p_{1}$ and advertising interval $z$ to maximize its overall expected profits in both periods: $\Pi=p_{1} D_{1}\left(z, p_{1}\right)+E_{q}\left(p_{2} D_{2}\left(p_{2}\right)\right)-A(z)$. In the Benchmark case, the firm solves

$$
\left(z^{0}, p_{1}^{0}\right)=\underset{z, p_{1}}{\arg \max } \Pi=\underset{z, p_{1}}{\arg \max } p_{1} z\left(1+\beta-z^{e} \tau-p_{1}\right)+p_{2}^{0}\left(1+\beta-\tau-p_{2}^{0}\right)-A(z) .
$$

Taking the first-order condition with respect to $p_{1}$, and plugging in the value for $p_{2}^{0}$, yields

$$
p_{1}^{0}=\frac{1+\beta-z^{0} \tau}{2}
$$

Taking the first-order condition for the advertising interval $z$, and substituting in $p_{1}^{0}$, yields

$$
\left(\frac{1+\beta-z^{0} \tau}{2}\right)^{2}=A^{\prime}\left(z^{0}\right) .
$$

\footnotetext{
${ }^{12}$ This conclusion holds even if the firm in the Benchmark case somehow obtains information on quality at the end of period 1 (say by conducting market research on first-generation buyers). Specifically, the firm could not use this information strategically to influence consumers' beliefs on quality as a separating equilibrium would not exist. This is because a low quality firm could always mimic a high quality firm as they are otherwise identical. Said differently, the high quality firm does not have an advantage it can exploit to price in a way that signals its higher quality to consumers and deter the low quality firm from mimicking.
} 
Equations (6) and (7) provide an implicit solution for $\left(p_{1}^{0}, z^{0}\right)$. As discussed following the derivation of period 2 demand functions (4) and (5), the expected demand size (as a function of price) is identical across informational cases. It is therefore not surprising that (6) remains unchanged in the different cases. Furthermore, the first-order conditions with respect to advertising are identical for the Benchmark and Reviews settings. This is because period 2 demand in both cases is independent of the advertising interval the firm selects. Intuitively, armed with Reviews second-generation consumers extract all the information about the product $(q$ and $y)$ regardless of the advertising interval size, leading to an independence between the choice of the ad interval and period 2 demand. In the Benchmark case, the reason for independence is in some sense opposite: regardless of the advertising interval selected, second-generation consumers do not learn any information about the product for the selection of $z$ to matter.

However, in the AR case we have

$$
\left(\frac{1+\beta-z^{A R} \tau}{2}\right)^{2}-\tau \frac{(1+\beta-\tau)}{2}=A^{\prime}\left(z^{A R}\right) .
$$

Thus, the advertising first-order condition in the AR case introduces an additional term, $-\tau \frac{(1+\beta-\tau)}{2}$, reflecting the fact that the firm takes into account that second-generation consumers will form an expectation of quality based on the average rating they observe $\mu_{1}$ (per (3)), which in turn will depend on the firm's selection of $z$.

The following proposition provides a comparison of the three UGC cases in terms of the implications for advertising and pricing strategies in equilibrium.

Proposition 1 In period 1, the optimal advertising interval and price are the same in the Benchmark and Reviews cases. In the AR case, the optimal advertising interval is smaller and the price is higher than in the other cases. That is, $z^{R}=z^{0}>z^{A R}$ and $p_{1}^{R}=p_{1}^{0}<p_{1}^{A R}$. In period 2, the optimal price is the same in the Reviews and $A R$ cases and increases in realized quality. The optimal price in the Benchmark case is independent of quality and satisfies $p_{2}^{0}<p_{1}^{0}$.

Proposition 1 reveals that the firm advertises to a smaller set of consumers in the AR case than in the other two information settings. The reason for this intriguing result is as follows. Given the average rating they observe, second-generation consumers use their belief with respect to the ad interval (i.e., $z^{e}$ ) to make an inference regarding quality (i.e., $q^{e}$ ). The firm takes as given consumers' beliefs when making its advertising decision (since consumers 
can't observe its choice of $z$ to alter their beliefs). For any value of $q$, a decrease in $z$ means that first-generation consumers whose ideal point is closer to the product's position will be the ones to buy. This, in turn, would lead to an increase in the average rating of first-generation buyers, and thus to an increase in second-generation consumers' estimate of $q$. Said differently, since second-generation consumers do not observe the distribution of locations and ratings but rather only the average rating $\mu_{1}$, they cannot fully separate out whether an increase in the observed average rating is due to the quality turning out to be high ex-post or due to buyers with better matching tastes rating the product. Consequently, they would attribute part of an increase in $\mu_{1}$ to quality, which increases their expected net utility for the product at any consumer location and hence their propensity to buy it. This introduces an incentive for the firm to decrease $z$. This incentive is absent in the Benchmark and Reviews cases because, as as explained earlier, period 2 demand is independent of the advertising interval chosen. Note that, in equilibrium, consumers should hold correct beliefs about the advertising interval $\left(z^{e}=z\right)$ in the AR case. Yet, as we show in the proof, the only beliefs $\left(z^{e}\right)$ that can work for the firm to not want to deviate from choosing $z^{A R}=z^{e}$ in equilibrium are such that $z^{A R}<z^{R}=z^{0} \cdot{ }^{13}$

As for the results on pricing, the finding that the period 1 price is higher in the AR case is directly linked to the smaller ad interval chosen. Because the firm focuses on consumers that are closer to the product's position, it reaches potential buyers who, on average, value the product more. In period 2, the firm and consumers glean the product's quality from available buyer generated content in both the AR and Reviews cases. As one might intuit, as the quality level is found to be greater the firm seeks to price higher, which explains the relationship between $p_{2}^{A R}, p_{2}^{R}$ and $q$ described in the proposition. In the Benchmark case, given that consumers do not learn the actual quality they must use its expected value (of 1), hence the firm's price is independent of quality (see also footnote 12). We note that because in period 2 all consumers are aware of the product, in all cases the firm has an incentive to lower price to maximize profits. Thus, price decreases in the Benchmark case and in expectation it decreases in the other cases as well $\left(E\left(p_{2}^{A R}\right)<p_{1}^{A R}, E\left(p_{2}^{R}\right)<p_{1}^{R}\right)$; although in the AR and Reviews cases, if $q$ is revealed to be sufficiently high, the price set in period 2 can exceed that of period $1 .{ }^{14}$

\footnotetext{
${ }^{13}$ If consumers in the AR case were to hold beliefs that the ad interval is the same as in the Reviews or Benchmark cases, then the firm would have an incentive to deviate to a smaller ad interval; hence those beliefs would not work in a PBE. The equilibrium ad interval we find, which is smaller than in the other two cases, is such that taking it as reflecting consumer beliefs the firm has no incentive to choose a different ad interval size. Hence, expectations are fulfilled and the firm is acting in its best interest.

${ }^{14}$ The marketing literature on new product diffusion also finds varying dynamic pricing patterns. However,
} 


\subsubsection{Average Rating Dynamics}

The findings regarding firm behavior in each of the informational cases yield a set of testable empirical implications when tracking the evolution of average ratings across periods. We denote by $\mu_{2}^{0}, \mu_{2}^{A R}, \mu_{2}^{R}$ the average ratings of second-generation buyers in the Benchmark, AR, and Reviews cases, respectively. It is straightforward to establish that: ${ }^{15}$

$$
\mu_{2}^{0}=\frac{1-p_{2}^{0}-\tau+(2 q-1) \beta}{2}, \mu_{2}^{A R}=\frac{1+\beta q-\tau-p_{2}^{A R}}{2}, \mu_{2}^{R}=\frac{3\left(1+\beta q-p_{2}^{R}-\tau\right)^{2}+\tau^{2}}{6\left(1+\beta q-p_{2}^{R}-\tau\right)}
$$

Comparing these average rating expressions we establish that

Proposition 2 The average rating dynamics in the three informational cases are:

1. Benchmark: the average rating of second-generation buyers is always lower than that of first-generation buyers.

2. AR: there exists a cutoff value $\bar{q}^{A R}\left(\bar{q}^{A R} \leq 1\right)$ such that the average rating of secondgeneration buyers is higher than that of first-generation buyers iff $q<\bar{q}^{A R}$.

3. Reviews: there exists a cutoff value $\bar{q}^{R}$ such that the average rating of second-generation buyers is higher than that of first-generation buyers iff $q<\bar{q}^{R}$. Moreover, $\bar{q}^{R}>\bar{q}^{A R}$.

Proposition 2 reveals that as more information is transferred across generations it is more likely for average ratings to increase. In particular, when there is no consumption-related information passed on, per the Benchmark case, the average rating the product receives will unequivocally go down over time. This occurs because in period 1 only consumers close to the product's position were made aware of the product and hence derived relatively high net utility upon purchase. In period 2, all consumers are aware of the product and thus anyone along the circle with non-negative expected utility purchases. This results in many consumers with lower valuations purchasing in period 2, some of whom may actually end up deriving negative

the mechanisms therein pertain to consumers' price sensitivity and/or discounting the future. For example, Bass et al. (1994) find that when consumers are highly price sensitive and/or uncertain about the future (high level of discounting), then lowering the price over time is worthwhile as a means to accelerate diffusion; otherwise it can make sense for the firm to increase price initially. Nair (2019) summarizes findings that a desire to initially cater to high-valuation customers can prompt a price skimming strategy (high followed by low price).

${ }^{15}$ Formal derivations of these expressions can be found in the Technical Appendix. In the Benchmark case, though no UGC is transferred, one can still compute each generation's average net utility and thus compare average ratings. Firms also commonly conduct satisfaction surveys, which provide a sense of this metric. 
utility ex-post (as second-generation consumers do not know the product's location nor can they infer $q$ ). Hence the declining pattern for average ratings in the Benchmark case. At the other extreme, per the Reviews case, there can be a scenario where average ratings increase across generations and, somewhat surprisingly, this occurs when the quality of the product is actually revealed to be low. Here, second-generation consumers know the location $y$ and quality $q$ of the product. This information allows those consumers who would get negative utility to avoid purchasing altogether. If the quality of the product turns out to be low enough $\left(q<\bar{q}^{R}\right)$, many first-generation consumers who were made aware of the product would have purchased the good (given their ex-ante expectation that $q=1$ ) only to be disappointed after their consumption experience. This dynamic will be reflected in low ratings in period 1, but higher ratings in period 2. In the AR case, second-generation consumers correctly infer $q$ in equilibrium, therefore once again it is possible for ratings to increase across periods. However, this occurs for a smaller range of qualities than in the Reviews case for two reasons: (a) the smaller advertising interval in the AR case (per Proposition 1) implies a relatively higher average rating for first-generation buyers, and (b) because in the AR case second-generation consumers cannot infer the product's position $(y)$, period 2 buyers are uniformly spread around the circle (vs. concentrated around the product's location in the Reviews case), which leads to a lower period 2 average rating.

An empirically relevant implication of Proposition 2 compares the average ratings in the $\mathrm{AR}$ and Reviews cases in both periods, revealing an intriguing reversal.

Corollary 1 For any $q$, the average rating in period 1 is higher in the AR case than in the Reviews case and the reverse ordering holds in period 2.

Figure 1 depicts the evolution of average ratings across periods. As explained, the average rating in period 1 will always be higher in the AR case given the advertising and pricing choices (per Proposition 1). In period 2, average ratings will depend on realized quality (per Proposition 2). Specifically, the right-most panel reflects the case whereby the realized quality $(q)$ is relatively high. In this setting, in both the AR and Reviews cases the period 2 average rating will decline. Notwithstanding, because second-generation consumers make more informed decisions in the Reviews case (with buyers concentrated around the product's location) the netutilities will decline less precipitously than in the AR case. In the middle panel of Figure 1, the realized quality is between the two cutoff values $\bar{q}^{R}$ and $\bar{q}^{A R}$. Thus, the average rating will increase in the Reviews case but decrease in the AR case. In this instance, not only is 
Figure 1: Average Rating Dynamics Across Periods as a Function of Realized Quality
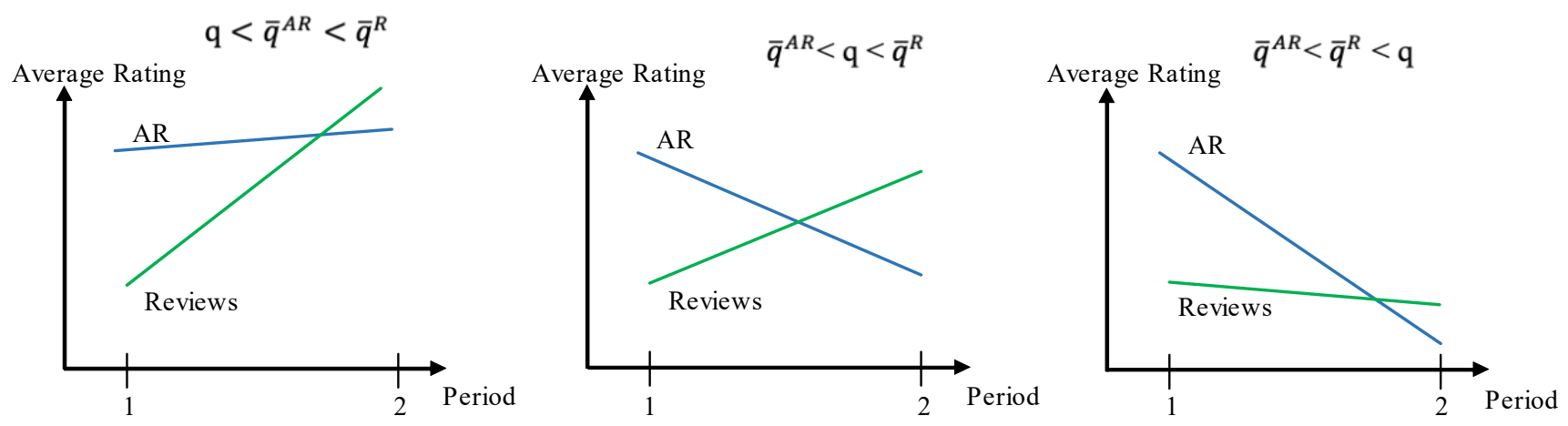

the ordering flipped over time but also the trend differs. Lastly, in the left-most panel realized quality is low, hence average ratings increase in both the AR and Reviews cases. Yet we would still have $\mu_{2}^{A R}<\mu_{2}^{R}$, given that fully informed second-generation buyers in the Reviews case are more concentrated around the product's location and derive greater net utility.

\subsubsection{Welfare: Firm Profits and Consumer Surplus}

In this section, we derive implications for firm profits and consumer surplus. We show that whether expected profits are highest in the AR or Reviews case depends on how costly advertising is, as well as on consumer sensitivity to product match and quality valuation. Using equilibrium values for the prices and advertising decisions, and recalling that $\Pi^{A R}$ and $\Pi^{R}$ denote expected profits in the AR and Reviews cases respectively, we have

Proposition 3 Expected consumer surplus is largest in the Reviews case. Expected profits can either be highest in the Reviews case or in the AR case. In particular, $\Pi^{R}>\Pi^{A R}$ if advertising is sufficiently costly $\left(A^{\prime}(z)\right.$ is large), consumers are sufficiently sensitive to the product match ( $\tau$ is large), or consumers do not value quality sufficiently ( $\beta$ is small). On the other hand, $\Pi^{R}<\Pi^{A R}$ if advertising is sufficiently cheap ( $A^{\prime}(z)$ is small), consumers are sufficiently insensitive to the product match ( $\tau$ is small), or consumers sufficiently value quality ( $\beta$ is large).

Both consumer surplus (CS) and expected profits are higher in the Reviews than in the Benchmark case. To see why, recall that the firm sets the same advertising interval and period 1 price in both instances (per Proposition 1). Therefore, the firm's period 1 profit and CS are identical in these cases. In period 2, CS is higher in the Reviews case because consumers are 
able to make purchase decisions based on the product's revealed quality and location. The firm is able to capture some of this additional value and translate it into greater profits by adjusting the price to reflect the product's quality, which is revealed over time. In the Benchmark case, by contrast, second-generation consumers do not know the product's location nor do they learn its quality, and hence act based on its expected value, i.e., as if $q=1$. Thus, the firm is limited in its ability to benefit from adjusting price in period 2 (only accommodating the greater awareness level by lowering price).

That CS is higher in the Reviews than in the AR case also follows a simple logic. In period 1, the advertising interval is smaller and the price is higher in the AR case compared to the Reviews case. As a result, period 1's CS is the lowest in the AR scenario. In period 2, CS is higher in the Reviews case because under AR consumers do not have information on product location yet face the same price in both instances.

In understanding the intuition for the expected profit results described in Proposition 3, one should recognize that in all information structures first-generation consumers must form a belief over the firm's advertising interval choice and this introduces a profit distortion. To see why, suppose the firm could credibly convey its choice of $z$ to consumers. In that case, the firm would choose a level $z=z^{*}$, where $z^{*}$ is the solution to the FOC: $\left(\frac{1+\beta-z^{*} \tau}{2}\right)^{2}-z^{*} \tau\left(\frac{1+\beta-z^{*} \tau}{2}\right)=A^{\prime}\left(z^{*}\right)$. This "ideal" advertising interval would lead to maximal expected profits for the firm. Now assume that consumers cannot observe the firm's actual choice yet hold beliefs that the firm will select this ideal ad interval, i.e., $z^{e}=z^{*}$. But then, in all information settings, the firm has an incentive to increase $z$. This is because the additionally targeted first-generation consumers, on average, think they are closer to the product's location than they actually are; leading to greater period 1 revenues for the firm. Of course, in equilibrium beliefs must be correct, but the only beliefs that can work result in a PBE with an advertising interval that is larger than what would have been ideal for the firm. For the Benchmark and Reviews cases, this is the only distortion in the firm's maximization problem. In the AR case, however, there is another force linked to period 2 revenues. As explained in connection with Proposition 1, the firm in the AR case has an incentive to decrease its advertising interval to bump up the average rating and hence the second-generation quality inference, which would lead to greater period 2 revenues. That is, in the AR case, the firm wishes to expand its ad interval to boost period 1 revenues and to contract its ad interval to boost period 2 revenues. To summarize, the equilibrium advertising interval in the Benchmark and Reviews cases is always too large relative to the 
Figure 2: Expected Profits in the AR and Reviews Cases: Low vs. High Advertising Costs

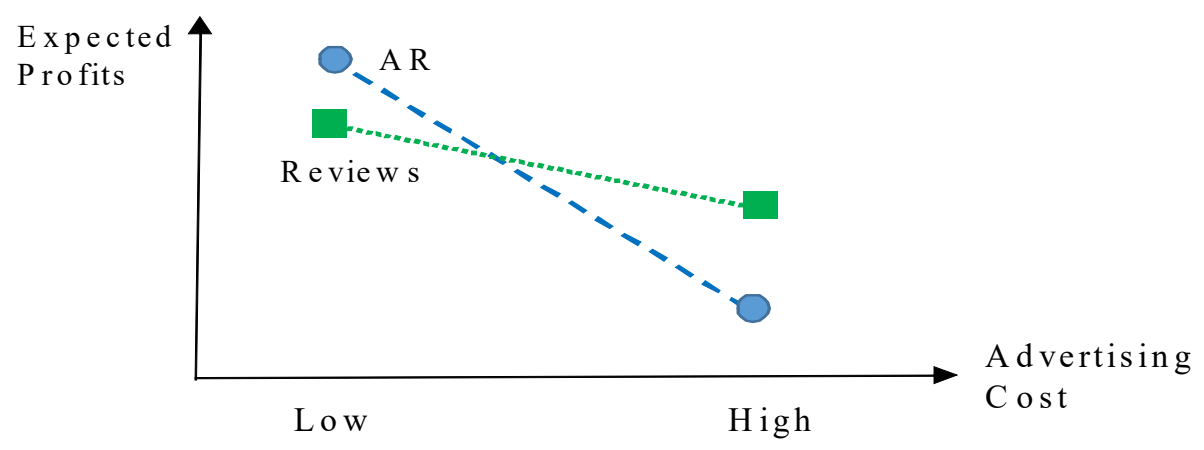

ideal interval $\left(z^{*}\right)$, whereas it can be too large, too small, or just about right, in the AR case but is always lower than in the Benchmark and Reviews cases due to the second incentive.

The relative proximity of $z^{R}$ vs. $z^{A R}$ to the ideal level $z^{*}$ determines in which information setting equilibrium expected profits will be greater. When advertising is costly (which occurs if $A^{\prime}(z)$ is large) or when the return on advertising in terms of generating extra demand is low (which occurs when $\tau$ is large or $\beta$ is small), the "ideal" advertising interval is small to begin with and forces that prompt increasing the ad interval are relatively weak whereas forces that prompt decreasing the ad interval are relatively strong. As a result, in equilibrium the advertising interval the firm chooses ends up being very close to the "ideal" level $z^{*}$ in the Reviews case $\left(z^{R}\right)$, and much smaller than $z^{*}$ in the AR case $\left(z^{A R}\right)$. The reverse holds when advertising is either cheap (small $A^{\prime}(z)$ ) or the return on it in terms of generating demand is high (small $\tau$ or large $\beta$ ), in which case $z^{A R}$ is closer than $z^{R}$ to the ideal level $z^{*}$ in equilibrium.

To illustrate, Figure 2 depicts the relationship between expected profits in the AR and Reviews cases as a function of advertising costliness. As can be gleaned, expected profits in the AR case can be higher or lower than in the Reviews case (similar plots can be constructed for the sensitivity to product match and to quality valuation).

\subsection{Robustness Checks and Extensions of the Basic Model}

In this section, we discuss relaxing several of the basic model's key assumptions and present a number of extensions. We seek to examine how the main findings, per Propositions 1-3, hold up under these modified settings and whether new findings emerge. When relevant, detailed proofs of these robustness checks and extensions are given in the Technical Appendix. 
Dynamic Market Size In the basic set up, we assumed that each generation of consumers was of the same size, normalized to one, and lived for one period. We discuss several ways that these assumptions can be relaxed (with full details in the Technical Appendix). First, we analyzed an alternative setup whereby first-generation consumers who were not made aware of the product in period 1 (i.e., were outside of the ad interval) remain in the market. These consumers realize in period 2 that they were not targeted in period 1 , hence they can make some inference on their expected distance to the product's position. We find that the main results from the basic model continue to hold: the ad interval is smallest and period 1 price is highest in the AR case (Proposition 1), and average ratings in the AR and Reviews cases can exhibit the ordering reversal as in Corollary 1. One new nuanced finding emerges from this analysis: the ad interval in the Reviews case is smaller than in the Benchmark case (so the ordering is $z^{A R}<z^{R}<z^{0}$ instead of $z^{A R}<z^{R}=z^{0}$ ). This occurs because the firm in the Reviews case has an incentive to shift some of the demand to period 2 and benefit from adjusting price.

Second, while maintaining the assumption that each generation of consumers lives for only one period, we analyzed a setting where the size of the second-generation cohort was allowed to vary (i.e., relaxing the normalization to one in period 2). As one might expect, the firm in the AR case has a greater incentive to reduce the ad interval and increase the period 1 price as the size of the second-generation cohort increases. Notwithstanding, all our results are qualitatively robust to this extension. It is worth noting that robustness holds even if the size of the second-generation cohort is much smaller than that of the first-generation cohort.

Discrete Average Ratings In characterizing the information second-generation consumers have at their disposal in the AR and Reviews cases, we assumed that the exact value of the average net utilities of first-generation buyers was available. This resulted in a one-to-one invertible mapping from average rating $\left(\mu_{1}\right)$ to quality $(q)$. However, on some platforms, e.g., Yelp!, average ratings are only displayed in discrete intervals. To examine the robustness of our findings to this possibility, we solved a setup where second-generation consumers do not observe $\mu_{1}$ directly but instead receive a signal $\widetilde{\mu} \in \mathcal{M}$, where $\mathcal{M}$ is a partition of $\mu_{1}$ into $n$ disjoint average rating intervals with respective expected means $\mu_{1,1}, \mu_{1,2}, \mu_{1,3}, \ldots \mu_{1, n}$. Here, $\mu_{1, m}$ is the signal received if $\mu_{1}$ belongs to the interval with mean $\mu_{1, m}$. This can capture, for example, Yelp's half-star system with $n=9$, so that $\mu_{1,1}$ is 1 star, $\mu_{1,2}$ is 1.5 stars, $\mu_{1,3}$ is 2 stars, etc. We show in the Technical Appendix that all aspects of Proposition 1-3 continue to hold 
qualitatively. This suggests that, even if average ratings are only cardinally informative, the firm has an incentive to restrict advertising and increase first-period price in the AR case, with corresponding implications as in the basic set up for rating dynamics and expected profits. ${ }^{16}$

\section{Manipulation, Bias, and Informativeness of User-Generated Evaluations Our work-} ing assumption in modeling user-generated ratings and reviews was that: the firm did not filter or influence the information presented, buyers reported truthfully, ratings/reviews constituted a representative (unbiased) sample of buyers, and all reviews posted contained meaningful information with all second-generation consumers attending to/absorbing this information. However, one might wonder how our findings would be affected if these assumptions were violated. ${ }^{17}$

Consider first the situation of a firm trying to affect subsequent consumer perceptions by removing unfavorable UGC. Specifically, let the firm designate a rating threshold $\kappa$ such that all evaluations (ratings in the AR case and ratings+reviews in the Reviews case) below this threshold are removed from the platform prior to the arrival of second-generation consumers. For example, on a 5-star scale removing all UGC associated with 1 or 2 stars (i.e., $\kappa=3$ ). We can show that, as long as $\kappa$ is not too high and consumers are rational, all our results continue to hold qualitatively. This is because consumers can still use the distribution of ratings above the threshold, along with their correct expectations with respect to $\kappa$, to infer the true average rating and hence back out quality. Moreover, in the Reviews case, assuming the firm does not alter the actual comments, consumers can use the information contained in the remaining reviews to infer product location. However, if the threshold is very close to the upper bound of the rating scale, it becomes impossible for second-generation consumers to back out the true average rating in the AR case and the firm effectively behaves as in the Benchmark case.

Next, consider the case in which the UGC is biased. The extant literature (see, e.g., Schoenmuller et al., 2020; Brandes et al., 2020, and references therein) has surfaced several reasons for such a bias, including various forms of selectivity whereby buyers with extremely positive or negative experiences have a greater tendency to rate/review (e.g., due to social utility gained from posting extreme reviews and reluctance to exert effort to transmit mediocre evaluations that are perceived to be less diagnostic). Once again, if second-generation consumers under-

\footnotetext{
${ }^{16}$ One can also consider a scenario where first-generation buyers provide ratings in discrete intervals ( 1 star, 2 stars, etc.), but the average of these discrete ratings is presented to second-generation consumers as a continuous value. In that case, with a sufficient number of buyers, the expected value of the average rating will converge to the expected mean of the net utilities. Hence, our analysis from the basic setup would continue to hold.

${ }^{17}$ The analyses of the extensions described below are available from the authors upon request.
} 
stand the nature of the bias (e.g., the percentage of those with each experience type that are expected to post an evaluation) and this bias is not too extreme (e.g., only those with a 5-star rating ever post), then consumers should be able to back out the true average rating. Otherwise, second-generation consumers in the AR case will treat the average rating as uninformative and the analysis collapses to the Benchmark case. Notwithstanding, in the Reviews case consumers are still able to extract product location and quality information from the comments posted and hence the conclusions from that analysis continue to hold.

Lastly, consider the case where all buyers leave reviews in addition to ratings, but the effective information transferred across generations is only "partial" - either because a fraction of the reviews are meaningless or because a fraction of second-generation consumers don't bother reading them. The former scenario could arise, for example, due to the platform's policy (requiring reviews to accompany ratings vs. making them optional), which could cause some buyers to write irrelevant comments. The latter scenario could arise due to time or motivation constraints (e.g., the statistics presented in the Introduction suggest that consumers often only attend to ratings). One can adjust our model to accommodate partial information transfer in the following way. In the Reviews case, let all second-generation consumers see the average rating, but assume that only a fraction $\lambda \in[0,1]$ of them absorb relevant information from the reviews. $^{18}$ It is immediate that $\lambda=0$ corresponds to the $\mathrm{AR}$ case and $\lambda=1$ to the Reviews case. We can thus think of second-generation consumers as divided into two circles of different mass (of size $\lambda$ and $1-\lambda$, respectively). Since the firm cannot identify which consumers are informed, it sets the same price and advertising interval for both circles. Consequently, the firm's maximization problem in effect puts a weight of $\lambda$ on the Reviews case profit function and a weight of $(1-\lambda)$ on the AR case profit function. The solution is smooth and monotone in $\lambda$, such that any comparison of firm actions and expected profits between two settings with values $\lambda_{1}$ and $\lambda_{2}\left(\lambda_{1}<\lambda_{2}\right)$ will correspond to a comparison between a "relatively AR" case and a "relatively Reviews" case, with qualitatively similar findings per Propositions 1-3.

Firm Knowledge of Product Location The firm in the basic set-up knows the exact location of the product (its "positioning") at the start of the game. Although we justified this assumption and provided several examples, one might wonder how our findings change if it is

\footnotetext{
${ }^{18}$ Thus, $\lambda$ either captures the fraction of second-generation consumers who: 1) sampled reviews that are informative or 2) actually read the reviews. Also assume that the realization of who gains relevant information from reviews is independent of each consumer's location and idiosyncratic product utility.
} 
relaxed. To examine this issue, one can envisage a setup where the firm only knows a range along the circle within which the location of a new product falls. There are two cases to analyze depending on whether the range of uncertainty is smaller or larger than the advertising interval the firm chooses when it knows the location precisely. In the former case, the firm would select an ad interval that includes the suspected location range, using the mean of the distribution as the center of this interval. It is fairly straightforward to show that all our findings per Propositions 1-3 continue to qualitatively hold in this modified setup, as the same set of firm incentives and consumer behavior are relevant. However, when the range of uncertainty is large, say the entire circle, the results qualitatively follow the situation where the firm cannot target its advertising. In that case, we might consider another form of advertising, whereby the firm communicates to all consumers and selects the ad intensity; see the next extension for the implications of this formulation.

Ad Intensity The firm in the basic model selects the interval over which to advertise in period 1 , with all targeted consumers in the interval becoming aware of the product. An alternative structure would be to assume that the firm advertises to all consumers and sets ad intensity, i.e., the probability that a consumer at a given location becomes aware of the new product. If the firm can vary the (costly) intensity along the circle, say by choosing between two levels - High (a consumer becomes aware with probability 1) and Low (a consumer becomes aware with probability $a, 0 \leq a<1$ ) - we expect all our findings to continue to hold. This is because the firm will have an incentive to advertise High over a smaller interval around the product location in the AR case compared to the Reviews and Benchmark cases; with all the results on rating dynamics and expected profits carrying through. This logic can be extended to any arbitrary number of ad intensity levels (with higher levels more costly to execute).

Consider alternatively that the ad intensity the firm selects applies equally to all consumers along the circle. This can be due to several reasons, for example: (1) limited advertising technology, i.e., the firm cannot single out segments that will receive greater or lower ad levels, or (2) as suggested at the end of the previous extension, the firm has no information on the product's position and thus has no preference to advertise in one location vs. another. In this restricted scenario, the results in Proposition 1 are qualified. In particular, prices and ad levels are the same across the different information structures in period 1. Yet rating dynamics still exhibit some of the patterns in Proposition 2. Details are provided in the Technical Appendix. 
Distribution Assumptions The basic set up has three product-related quantities for which a uniform distribution was assumed: quality $q \sim U[0,2]$, location $y \sim U[0,4]$, and idiosyncratic consumer valuation $\alpha_{i} \sim U[0,1]$. Although these are fairly standard assumptions, which have been used in prior literature (e.g., Kuksov and Xie, 2010, with respect to quality) and simplify the analysis, the reader may wonder whether the findings presented are sensitive to the distributional form.

With respect to quality, we analyzed the basic model using a general continuous distribution function $Q$ over $[0,2]$ with mean 1 . We find that all the results reported in Propositions 1-3 are robust. With respect to the base consumer valuation $\alpha_{i}$, we similarly checked a general continuous distribution $F$ over $[0,1]$. Here we establish that the conclusions from Proposition 1 all continue to hold. Yet showing that the other propositions hold requires more structure on the distribution function. These analyses are presented in the Technical Appendix.

With respect to product location, recall that the distribution of $y$ affects how consumers infer horizontal product fit (absent any other information). While it is analytically intractable to solve for a general distribution function for $y$, we did analyze an alternative model whereby aware consumers, i.e., those who received an ad in the first period and all consumers in the second period, perfectly know the product's location (in some sense a point mass distribution for $y$ ). We show that the results in Propositions 1-2 continue to hold under this structure. As for Proposition 3, we find that expected profits are always highest in the Reviews case, in contrast with the basic model where expected profits could be highest in the AR case (see the Technical Appendix for full details). By continuity, these conclusions hold for any general distribution of $y$ over a small arc around the expected mean location.

Alternative Pricing Structures A feature of the basic set-up is that the firm is able to set a different price in each period. This characteristic is reflective of many markets. However, there are industries for which this structure is not well suited. For instance, when considerable consumer backlash is triggered by a subsequent price change. There may also be costs or time delays associated with adjusting prices, such as updating catalogs, signage, etc. (also known as menu costs; see Mankiw, 1985), or there may be contractual rigidities that prevent frequent price shifts (also known as staggered contracts; see Taylor, 1980). Such factors can deter firms from adjusting prices. Moreover, in some markets the firm may not have much control in setting prices, either because of regulation or long-term agreements (e.g., a studio is not able to affect 
theater ticket prices for a movie irrespective of its quality or production cost). On the demand side, we assumed that second-generation consumers were able to observe the price history, i.e., they knew both $p_{1}$ and $p_{2}$, in all information settings. While this is realistic in the Reviews case, as the price paid can be backed out given the other information communicated, this may be a strong assumption in the AR and Benchmark cases.

In the Technical Appendix, we analyze three different pricing structures in which: (1) prices are exogenous, (2) the firm sets one price for the entire game (i.e., the period 1 price applies to period 2), (3) the firm sets price in each period but second-generation consumers do not observe the period 1 price. We show that our main findings carry over to model variations (1) and (2): the firm advertises less in the AR case than in the Benchmark and Reviews cases, which drives much of the subsequent results on rating dynamics and welfare. One exception is that in scenario (2) the single price set in the AR case can be lower than the Reviews' price.

When price history is not observable (scenario (3)), the firm's actions are unchanged in the Benchmark and Reviews cases, yet are affected in the AR case. Note that here, secondgeneration consumers must form an expectation on $p_{1}$, and the firm has an incentive to lower the period 1 price in order to bump up the average rating of first-generation buyers. Hence, the firm not only selects a smaller ad interval but also a lower price in equilibrium compared to the observable price history setting (and this price may even be lower than the Benchmark and Reviews case prices). As a result of these actions, expected profits in the AR case are lower when the price history is unobserved compared to the basic model. Otherwise, similar qualitative findings as in the main model hold when comparing the Reviews and AR cases, i.e., the AR ad interval will be smaller (as in Proposition 1) and the average rating dynamics will exhibit the same patterns (as in Proposition 2).

Consumer Uncertainty about Firm Type In the basic model, the firm's incentive to affect first-generation UGC is clearly linked to the fact that the firm cares about future profits. However, if some firms are forward looking (patient) while others only care about short term profits (impatient), and this information is private, second-generation consumers must try to assess which firm they are facing in order to draw proper inferences.

To consider this situation, suppose that with probability 0.5 the firm is patient (as in the basic model), whereas with probability 0.5 the firm is impatient and chooses $p_{1}$ and $z$ to maximize period 1 profits only. An impatient firm would obviously select the same price and 
advertising level in all information structures. Hence, if the patient firm chooses a different price, this reveals its type (separating equilibrium) and the analysis follows that in the basic model. Another option for the patient firm is to mimic the price set by the impatient firm (pooling equilibrium). In this instance, consumers do not know which firm they are facing and, in equilibrium, their expectation of the advertising interval is an average of the intervals chosen by the patient and impatient firms. As a result, in the AR case consumers overestimate the quality of the patient firm (which still has an incentive to select a smaller ad interval) and underestimate the quality of the impatient firm. This effect will increase the expected profits of the patient firm and decrease those of the impatient firm. If pooling occurs in both the AR and Reviews cases, then the comparison of the patient firm's actions and expected profits across the two information structures is similar to that in the exogenous price case described above. ${ }^{19}$

\section{Incorporating Social Interactions}

In the basic model presented thus far, we assumed that all second-generation consumers are aware of the product. This was meant to reflect settings whereby once a new product has been in the marketplace for a full period, then either through WOM, observational learning, or media coverage, consumers in period 2 are apprised of its existence.

While this is a reasonable assumption, which allowed us to focus on the implications of various user-generated inputs available to second-generation consumers (average ratings or full reviews), one wonders how micro-modeling the inter-generational awareness process might affect our findings and whether new insights can be obtained. Furthermore, if indeed secondgeneration awareness is contingent upon some form of social contagion from past buyers, then one can expect demand in period 2 to be a direct function of the demand size in period 1. To explore these issues in greater depth, we now relax the assumption of "automatic" secondgeneration awareness and let it depend on social interactions with previous buyers.

Let second-generation consumer $j$ who is located at $x$ interact with a representative sample of first-generation consumers from a location chosen according to the following distribution: with probability $\eta$ these consumers are located at $x$, and with probability $(1-\eta)$ their location is drawn at random uniformly from around the circle. Consumer $j$ knows the general underlying sampling process. If any of the sampled first-generation consumers purchased the product, then

\footnotetext{
${ }^{19}$ Existence of these equilibria depends on model parameters; analysis available from the authors upon request.
} 
consumer $j$ becomes aware of it; otherwise she remains unaware. The parameter $\eta(0<\eta<1)$ thus captures to what extent a second-generation consumer is likely to be paired with a firstgeneration consumer who is exactly like her in terms of horizontal tastes- in other words the degree of "homophily." In particular, if $\eta \rightarrow 1$, consumer $j$ knows she is interacting with firstgeneration consumers that have the same location as she does, whereas if $\eta \rightarrow 0$, her interaction is with people she is entirely unsure about their preference along the circle.

Once a second-generation consumer is aware of the product, we can consider two ways to model how she gains more information about it. One option is to assume, as in the basic setup, that she has access to UGC from first-generation buyers, either in the form of an average rating or full reviews. The other option is to assume that an aware second-generation consumer learns exclusively from the first-generation buyers she is paired with. We concentrate here on presenting the results from the former setting, as it allows a clean comparison to the basic model setup and bears on understanding the impact of various user-generated inputs on firm behavior. We present results from analyzing the latter setting in the Technical Appendix and show that most of the findings are qualitatively similar.

Recall that in Section 3.2 we explained that the pricing structure does not have a major impact on the analysis of other variables of interest. Thus, for ease of exposition, we focus here on a setting where prices are exogenously given. This simplifies the derivations and allows highlighting how micro-modeling awareness through cross-generational interaction affects the firm's choice of advertising interval, welfare, and average rating patterns. We again solve for the $\mathrm{PBE}$ of the game and require that consumer expectations on unobserved firm decisions be fulfilled in equilibrium. All proofs for this section appear in the Technical Appendix.

\subsection{Demand}

It is easy to verify that the expressions for the demand and average rating of first-generation consumers carry over unchanged from the basic model of Section 3. Using superscript $N$ to denote the setup with social network interactions, we can thus write $D_{1}^{N}=D_{1}=z\left(1+\beta-z^{e} \tau-p\right)$, with the mean net utility (i.e., average rating) of buyers $\mu_{1}^{N}(z)=\mu_{1}(z)=\frac{1+\beta(2 q-1)-\left(2 z-z^{e}\right) \tau-p}{2}$.

The demand in period 2 depends on the inter-generation transmission of information. We denote by $D_{2}^{N-0}, D_{2}^{N-A R}, D_{2}^{N-R}$ period 2 demand in the Benchmark, Average Ratings (AR), 
and Reviews cases, respectively. In the Technical Appendix we establish that

$$
\begin{aligned}
& D_{2}^{N-0}(z)=z\left(1-p+\beta-\tau+\tau \eta-z^{e} \tau \eta\right), D_{2}^{N-A R}(z)=z\left(1-p+q^{e} \beta-\tau+\tau \eta-z^{e} \tau \eta\right) \\
& D_{2}^{N-R}(z)=z(1-p+q \beta-\tau+\tau \eta-z \tau \eta) .
\end{aligned}
$$

It is apparent from the expressions in (9) that the demand in period 2 differs across information structures. In particular, when no information is transmitted (Benchmark) demand is independent of quality. However, when information is transmitted, demand depends on either expected quality (AR) or actual quality (Reviews). Notice that with social interactions demand in period 2 in the Benchmark and AR settings also depends on $z^{e}$ through the term $-z^{e} \tau \eta$. This is because when second-generation consumers become aware of the product, their expected utility from purchasing it depends on how close they think they are to its position, which, in turn, depends on how likely they believe their awareness was generated by someone like them and how close, on average, that first-generation buyer is to the product's location.

In equilibrium, as expectations are fulfilled, $z^{e}=z$ and $q^{e}=q$, and we can write

$$
D_{2}^{N-0}(z)=z(1-p+\beta-\tau+\tau \eta-z \tau \eta), D_{2}^{N-A R}(z)=z(1-p+q \beta-\tau+\tau \eta-z \tau \eta)
$$

It is noteworthy that all the demand expressions have a multiplying factor of $z$, the advertising interval size. This is intuitive, as only first-generation consumers who were targeted with advertising could have purchased the good and serve as a source of awareness for secondgeneration consumers. Hence, it should not be surprising that, under all information structures, the advertising interval the firm selects is larger in the social-interaction model studied here than in the basic model. Put differently, because demand in period 2 directly depends on the size of the demand from period 1, the firm has an incentive to advertise more extensively in period 1 to generate a sufficient number of "social contagion agents".

It is further noteworthy that period 2 demand in all cases depends on the homophily parameter $\eta$. This is because greater homophily implies higher chances of becoming aware of the product from someone whose preferences are similar, which has implications for how close to the product's location one expects to be and thus for assessing net utility from purchase. 


\subsection{Advertising}

Solving for the optimal ad interval, we can state the following dependence on homophily.

Proposition 4 In the Benchmark and $A R$ cases, the advertising interval the firm selects increases in the homophily level. In the Reviews case, the advertising interval increases in the homophily level if advertising is sufficiently costly $\left(A^{\prime}(z)\right.$ is large), consumers are sufficiently sensitive to the product match ( $\tau$ is large), or consumers do not value quality sufficiently ( $\beta$ is small). Otherwise, the advertising interval in the Reviews case decreases in the homophily level.

The intuition for Proposition 4 is as follows. As homophily gets stronger ( $\eta$ increases), probabilistically more second-generation consumers who are at a smaller than average distance from the product are made aware of its existence. In the Benchmark and AR cases, aware second-generation consumers do not learn the exact location of the product, but rather draw an inference about their distance from it based on the ad interval they believe the firm selected $\left(z^{e}\right)$. Hence, given these beliefs, from the firm's point of view there is an incentive to increase the advertising interval to gain additional second-generation consumers who would think they are closer to the product than they actually are (as they don't observe the actual $z$ chosen). Of course, in equilibrium consumers' beliefs have to be correct. Yet the only beliefs that work to form a PBE and avoid deviation entail an ad interval that grows as homophily increases to account for the firm's incentive.

Intuition for the effect of homophily in the Reviews case is as follows. When advertising is relatively costly to execute, or when consumer sensitivity to product match/quality are such that the return on advertising spend is low, the ad interval the firm chooses is in the range $z^{N-R}<\frac{1}{2}$. Consequently, expanding the advertising interval increases period 1 demand in locations that are still relatively close to the product's position. Greater homophily, by definition, means that this action will increase awareness in period 2 at locations that are closer than average to the product. This is beneficial to the firm as more second-generation consumers will be willing to buy, and justifies the added advertising expenditure. The opposite occurs, however, when advertising is relatively cheap to execute or consumer sensitivity to product match/quality are such that the return on advertising spend is high; and the ad interval is hence in the range $z^{N-R}>\frac{1}{2}$. In this case, many second-generation consumers who are relatively far away from the product are made aware and may be unwilling to buy the product (since they know the product's exact location from reading the reviews and therefore how far it is from them). Thus, 
as homophily increases, the firm is better off contracting the interval and focusing on consumers in both generations who are closer to the product's position.

We now compare the advertising interval selected in the various information structures.

Proposition 5 The advertising interval chosen is the largest in the Benchmark case, and is larger in the Reviews than in the AR case: $z^{N-A R}<z^{N-R}<z^{N-0}$.

As in the basic model, the advertising interval in the AR case is smallest. However, Proposition 5 reveals a somewhat different ordering among the other two cases. Specifically, the reason that the advertising interval is the largest in the Benchmark case is as follows. Expanding the ad interval results in more aware consumers in period 2 who presume they are closer to the product than they actually are, because they rely on $z^{e}$ and not $z$ (which they do not observe) to assess their expected distance from the product's position. Hence, the firm has a strong incentive to increase $z$. Of course, in equilibrium it must be that $z^{e}=z$, but due to this incentive the only set of beliefs that can work leads to a larger ad interval than in the other cases. Note that although this incentive also exists in the AR case, there the firm has a strong countervailing force pushing it to reduce the size of the ad interval in order to cause aware second-generation consumers to bump up their assessment of quality (just as in the basic set up, as explained in connection with Proposition 1). In the Reviews case, by contrast, because second-generation consumers know the location and quality of the product with certainty, these incentives to increase/decrease $z$ are absent.

\subsection{Average Rating Dynamics}

Turning to how the social interaction process impacts average rating dynamics, we have

Proposition 6 In the Benchmark case, the average rating decreases across periods. In the AR and Reviews cases, the average ratings increase over time for a larger range of qualities in the social-interaction setting compared to the basic model.

The intuition for the first part of Proposition 6 follows a similar logic as in the basic model: with no information transferred, second-generation buyers have, on average, lower utility from the product than first-generation buyers. For the second part, we begin by noting that the period 2 average rating is higher in the social-interaction setting compared to the corresponding 
basic model values. The reason is that, for any positive level of homophily, the aware consumers in period 2 tend to originate from locations that are closer in proximity to the product's position than in the basic model. Furthermore, according to the observation made following the derivation of the period 2 demand functions ((9) and (10)), in all informational cases the advertising interval is larger in the social-interaction setting. This implies that in period 1 consumers who are farther away from the product's location are made aware of it. Thus, period 1 ratings tend to be lower in the social-interaction setting compared to the basic model. Taken together, the tendency with social interactions for lower first-generation ratings and higher second-generation ratings produce the pattern described in Proposition 6.

\subsection{Welfare: Firm Profits and Consumer Surplus}

The following proposition presents key properties of the expected profits.

Proposition 7 In the social-interaction setting, expected profits in all informational cases increase in homophily. Moreover, expected profits are lowest in the Benchmark case and highest in the $A R$ case. That is, $\Pi^{A R}>\Pi^{R}>\Pi^{0}$.

In all three information settings, greater homophily implies that second-generation consumers who become aware of the product are more likely to be similar in taste preferences to first-generation buyers (who are close to the product's position) and hence have a greater willingness to buy. This increases demand and thus profits.

The proposition reveals that, somewhat surprisingly, with social interactions expected profits are always highest in the AR case. This is because, in all information settings, the firm has an incentive to allow second-generation consumers to interact with more first-generation buyers in order to increase awareness. This is accomplished by expanding the advertising interval beyond the "ideal" level the firm would have chosen had advertising been observable (and which would result in maximal expected profits). But in the AR case, the firm also has an incentive to shrink the advertising interval in an effort to bump up the average rating (to affect second-generation quality inferences). Hence, one can show that the equilibrium advertising interval chosen by the firm under AR is closer to the ideal level, compared to the Benchmark and Reviews cases.

Finally, there are two forces affecting CS in the social-interaction setup: 1) a larger advertising interval increases CS, and 2) for a given advertising interval, when consumers have 
more information they make better purchase decisions and their surplus increases. From these observations and Proposition 5, it follows that $C S^{N-R} \geq C S^{N-A R}$.

\section{Concluding Remarks}

In this paper, our primary goal has been to study how a firm's strategic marketing decisions depend on the nature of customer-generated content available to prospective buyers. In particular, we developed a two-period model in which a firm introduces a new product and selects its advertising and pricing strategies. The ex-ante quality of the product is unknown, and a sub-set of consumers become aware of the product's existence when targeted by the firm's advertising. Consumers who purchased the product in the first period might be able to share their experience with consumers in the second period. We examined three information transfer structures: a Benchmark case where no evaluations are passed on; an Average Rating (AR) case where second-generation consumers only observe the average net utility of those who purchased; and a Reviews case where second-generation consumers observe the joint distribution of net utilities and taste locations of those who purchased.

The basic model we analyzed is one where all second-generation consumers become aware that the new product has been launched, reflecting settings where word of the product's existence has spread widely (e.g., through the media or observational learning). In this context, we first show that while the firm selects the same advertising interval in the Reviews and the Benchmark cases, its advertising scope in the AR case is more limited. The reason behind this result is that if consumers expect the firm in the AR case to select the same advertising reach as in the Reviews case, the firm has an incentive to deviate and target a smaller segment of consumers closer to the product's location. This deviation would increase the average rating seen by second-generation consumers who would infer a higher quality product; thus generating a greater profit for the firm. In equilibrium, the firm indeed selects a smaller ad segment, such that if consumers' beliefs are consistent with this level it does not wish to deviate. As a result of targeting consumers closer to the product's location in the AR case, the firm charges a higher price to take advantage of the greater willingness to pay.

We further presented results regarding the dynamics of average ratings. In particular, as customer-generated assessments become more informative, the average rating is more likely to increase over time. This is because when reviews are available, a second-generation consumer 
learns not only the product's quality but also its positioning and is able to make purchase decisions without any uncertainty; thereby avoiding the possibility of experiencing negative utility ex-post. In comparison, when only an average rating is available such learning of the product's position is not possible; hence some customers will end up being disappointed and reflect this in their rating. Combining this result with the different advertising strategies, whereby the firm in the AR case targets consumers closer to the product's position, leads to an interesting pattern: the average rating will be higher in period 1 in the AR case but higher in period 2 in the Reviews case. In addition, we characterized conditions for average ratings to decrease across generations. Interestingly, such a declining pattern happens when first-generation buyers discover that the product is of relatively high quality, and their UGC prompts more second-generation consumers to purchase, many of whom will have very low net utility, which will be reflected in their modest ratings.

We also analyzed the impact of the information structure on welfare. We find that expected profits may have a non-monotonic dependence on the amount of UGC conveyed. Specifically, if the marginal return on advertising spending is high - which occurs if advertising is cheap, consumers are insensitive to product fit, or consumers value quality sufficiently - profits will be highest in the AR case. This occurs because the firm's incentive to restrict the ad interval to affect second-generation consumer inferences counterbalances the incentive to choose a large ad interval to increase period 1 demand, leading to an ad level that is close to "ideal" (i.e., the level that would maximize profits had consumers been able to observe the actual advertising interval). By contrast, when the marginal return on advertising is low, the latter incentive is weak and the distortion from limiting the ad interval dominates, leading to lower expected profits in the AR case compared to the Reviews case.

We discussed a number of possible robustness checks and extensions to the basic setup (in Section 3.2). Moreover, we presented an alternative setting where awareness in period 2 is a function of social interaction across generations. Specifically, a second-generation consumer interacts with a sample of first-generation consumers whose location along the circle is either the same as her own, reflecting homophily, or randomly drawn. In this context, we find that the firm casts the widest advertising net when no information is transferred across generations (Benchmark case) and that, as in the basic setup, the smallest ad interval is in the AR case. Furthermore, as the degree of homophily increases the firm tends to advertise more extensively in the first period. This is primarily true in the Benchmark and AR cases because second- 
generation consumers don't know the product's exact position. Hence, if the first-generation consumers they interact with are more likely to be similar to them, they infer greater proximity to the product's location. This gives the firm an incentive to target additional consumers in period 1 to serve as social contagion agents, so that more second-generation consumers will presume they are closer to the product than they actually are. In equilibrium, beliefs have to be correct, hence to accommodate the firm's incentives the ad interval (and consumers' belief about it) increases in homophily. Lastly, with social interactions we find that expected profits always increase in the degree of homophily, attaining the highest level in the AR case. In other words, limited UGC in the form of an average rating benefits the firm.

\subsection{Managerial Implications}

Our findings have implications for firms, regulators, and platforms such as Airbnb. First, in terms of advertising strategy, firms that enable and require detailed comments (Reviews), compared to firms that only feature average ratings (AR), should expand their advertising program when launching a new product.

Second, with respect to pricing, companies that focus on soliciting and displaying only average ratings should consider initially pricing higher compared to when full reviews are available (and read). At the same time, with any form of user-generated evaluations, a company should be ready to adjust its price over time as market perceptions of quality are revealed.

Third, a key observation emanating from our analysis is that the amount of UGC transferred across generations can result in different average rating patterns over time. In particular, a new product should expect to have a higher average rating soon after its release in the AR case compared to when full Reviews are available. But that gap should diminish over time and even flip. Furthermore, there can be scenarios where more information transferred (Reviews) results in an increasing pattern for average ratings, whereas limited information (AR) results in a decreasing pattern - despite holding quality constant. So the firm needs to factor in how much information is transferred to draw the right conclusions about its product from average ratings data. Some empirical evidence we collected seems consistent with these implications. Specifically, we randomly sampled ten electronic products at Amazon, which up until recently had required review comments in addition to ratings when posting evaluations, ${ }^{20}$

\footnotetext{
${ }^{20}$ After many years, in late 2019 Amazon modified its policy to allow customers to leave only ratings, with review comments optional (Del Rey, 2020). Our analysis (till August 2019) spans a period where reviews were
} 
and at WalMart.com, which allows inputting just ratings (comments are optional and often left blank). We find that the average rating for the same sampled products is significantly higher in the first median of the data at Wal-Mart compared to Amazon. In addition, the average rating exhibits a significant declining trend at Wal-Mart but an increasing trend at Amazon (see the Technical Appendix for details of this analysis and a discussion of its limitations, particularly as they pertain to the quality of information transferred on these platforms).

Fourth, several firms and platforms (e.g., Open Table's mobile app) only solicit ratings from past customers, and present future consumers with the average rating across all respondents. ${ }^{21}$ Our analysis suggests that this strategy can make sense in environments where targeted advertising is relatively inexpensive to execute or consumers don't have strong horizontal preferences but highly value quality. Other firms (e.g., Best-Buy) encourage and even require detailed reviews rather than just ratings on their webpages - a strategy that can make sense when advertising is costly or when consumers care about product fit. One must also bear in mind the positive externality generated when a firm introduces a system for detailed reviews- consumer surplus is enhanced. Thus, regulators should consider motivating companies to create outlets for reviews and encourage prospective buyers to read them. The results also provide a rationale for certain platforms, such as Airbnb, to invest extensively in their review system- including highlighting informative reviews, prompting consumers to post reviews, making reviews easily searchable, and maintaining review integrity. By doing so, they may increase third-party sellers' profits (as presumably advertising is relatively difficult for them to execute) and consumers' surplus; thereby attracting many participants on both sides of the platform.

Lastly, in market contexts where consumers rely heavily on WOM from past buyers to become aware of innovations, the firm can benefit from advertising more extensively early on in the product lifecycle to produce social-contagion agents. This is especially true if "like-minded" consumers with a high degree of homophily are expected to connect with each other. Therefore, the practice of narrowly seeding only the most enthusiastic consumers for a product (in the sense that it more closely fits their tastes) may be misguided in some UGC settings. In addition, facilitating social interactions between like-minded consumers, say through friend-bring-friend incentives, coupled with displaying only the average rating can serve the firm well profit-wise.

still required on the platform.

${ }^{21}$ In many other cases, while detailed reviews are possible to get to eventually, the first screen a consumer is shown only reveals the average ratings of various options. Such is the case on Google Play with respect to apps and mobile games. So a consumer's initial reaction to an offering may only be based on the average rating. 


\section{Appendix}

Proof of Proposition 1. The proof follows from three lemmas (Lemmas 1-3), noting that, in equilibrium, $q^{e}=q$.

Lemma 1 In the Benchmark case, the equilibrium price and advertising strategies solve the following equations: $p_{1}^{0}=\frac{1+\beta-z^{0} \tau}{2}, p_{2}^{0}=\frac{1+\beta-\tau}{2},\left(\frac{1+\beta-z^{0} \tau}{2}\right)^{2}=A^{\prime}\left(z^{0}\right)$.

Proof. In period 2 the firm solves $p_{2}^{0}=\arg \max _{p} p\left(D_{2}^{0}(p)\right)$ or $p_{2}^{0}=\arg \max _{p} p(1+\beta-\tau-p)$, which leads to $p_{2}^{0}=\frac{1+\beta-\tau}{2}$. In period 1 the firm solves $\left(z^{0}, p_{1}^{0}\right)=\arg \max _{z, p} \Pi=p D_{1}(z, p)+E_{q}\left(p_{2}^{0} D_{2}^{0}\left(p_{2}^{0}\right)\right)$ $-A(z)=p z\left(1+\beta-z^{e} \tau-p\right)+E_{q}\left(p_{2}^{0}\left(1+\beta-\tau-p_{2}^{0}\right)\right)-A(z)$. Because $E_{q}\left(p_{2}^{0}\left(1+\beta-\tau-p_{2}^{0}\right)\right)$ is independent of $(z, p), p_{1}^{0}$ and $z^{0}$ are chosen to maximize period 1 profits. A first-order analysis and then substituting $z^{e}=z^{0}$ yields $p_{1}^{0}=\frac{1+\beta-z^{0} \tau}{2}$ and $p_{1}^{0}\left(1+\beta-z^{0} \tau-p_{1}^{0}\right)=A^{\prime}\left(z^{0}\right)$. Substituting in the first-order condition for $p_{1}^{0}$ we get $\left(\frac{1+\beta-z^{0} \tau}{2}\right)^{2}=A^{\prime}\left(z^{0}\right)$.

Lemma 2 In the AR case, the equilibrium price and advertising strategies solve the following equations:

$p_{1}^{A R}=\frac{1+\beta-z^{A R} \tau}{2}, p_{2}^{A R}=\frac{1+\beta q^{e}-\tau}{2},\left(\frac{1+\beta-z^{A R} \tau}{2}\right)^{2}-\tau \frac{1+\beta-\tau}{2}=A^{\prime}\left(z^{A R}\right)$. We have $z^{A R}<z^{0}$ and $p_{1}^{A R}>p_{1}^{0}$.

Proof. In period 2, the firm solves $p_{2}^{A R}=\arg \max _{p} p\left(D_{2}^{A R}(p)\right)$ or $p_{2}^{A R}=\arg \max _{p} p\left(1+\beta q^{e}-\tau-p\right)$. A first-order analysis yields $p_{2}^{A R}=\frac{1+\beta q^{e}-\tau}{2}$. In period 1 , the firm solves $\left(z^{A R}, p_{1}^{A R}\right)=\arg \max _{z, p} \Pi$ $=p D_{1}(z, p)+E_{q}\left(p_{2}^{A R} D_{2}^{A R}\left(p_{2}^{A R}\right)\right)-A(z)=p\left(z\left(1+\beta-z^{e} \tau-p\right)\right)+E_{q}\left(p_{2}^{A R}\left(1+\beta q^{e}-\tau-p_{2}^{A R}\right)\right)$ $-A(z)$. Note that $E_{q}\left(p_{2}^{A R}\left(1+\beta q^{e}-\tau-p_{2}^{A R}\right)\right)$ is independent of $p_{1}^{A R}$. Thus, the first-order condition for $p_{1}^{A R}$ yields $p_{1}^{A R}=\frac{1+\beta-z^{A R} \tau}{2}$. Because $q^{e}$ is only piece-wise differentiable in $z$, a first-order condition for $z$ over the entire range $[0,1]$ is not well defined. We deal with this in three steps. First, we derive a first-order condition for $z^{A R}$ for each of the three ranges over which $\frac{\partial q^{e}}{\partial z}$ is well defined. Second, we argue that an equilibrium value of $z^{A R}$ must solve the first-order condition in the middle range of the three, thus establishing a unique equilibrium candidate. Third, we show that there is no profitable deviation from the unique equilibrium candidate even outside the range covered by the first-order condition.

To begin, note that, $E_{q}\left(p_{2}^{A R}\left(1+\beta q^{e}-\tau-p_{2}^{A R}\right)\right)$ depends on $z$ through the effect of $z$ on $\mu_{1}$, which is an input for $q^{e}$ and thus also for $p_{2}^{A R}$. In particular, if $\frac{\partial E_{q}\left(p_{2}^{A R}\left(1+\beta q^{e}-\tau-p_{2}^{A R}\right)\right)}{\partial z}$ exists, by the 
Leibnitz rule it equals $E_{q} \frac{\partial E_{q}\left(p_{2}^{A R}\left(1+\beta q^{e}-\tau-p_{2}^{A R}\right)\right)}{\partial z}$. Developing this expression further we get that

$$
\begin{aligned}
& \frac{\partial E_{q}\left(p_{2}^{A R}\left(1+\beta q^{e}-\tau-p_{2}^{A R}\right)\right)}{\partial z}=E_{q} \frac{\partial\left(p_{2}^{A R}\left(1+\beta q^{e}-\tau-p_{2}^{A R}\right)\right)}{\partial z} \\
= & E_{q}\left(\frac{\partial\left(p_{2}^{A R}\left(1+\beta q^{e}-\tau-p_{2}^{A R}\right)\right)}{\partial p_{2}^{A R}} \frac{\partial p_{2}^{A R}}{\partial z}+\frac{\partial\left(p_{2}^{A R}\left(1+\beta q^{e}-\tau-p_{2}^{A R}\right)\right)}{\partial q^{e}} \frac{\partial q^{e}}{\partial z}\right) \\
= & E_{q}\left(\left(q^{e} \beta-\tau-2 p_{2}^{A R}+1\right) \frac{\beta}{2} \frac{\partial q^{e}}{\partial z}+p_{2}^{A R} \beta \frac{\partial q^{e}}{\partial z}\right)=\frac{1}{2} E_{q}\left(\left(q^{e} \beta-\tau+1\right) \beta \frac{\partial q^{e}}{\partial z}\right) .
\end{aligned}
$$

Notably, $\frac{\partial q^{e}}{\partial z}$ is well-defined in all but at most 1 point, which is determined by the value of $z-z^{e}$. At that point $q \in\{0,2\}$ and hence $\left(q^{e} \beta-\tau+1\right) \in\{1-\tau, 1-\tau+2 \beta\}$. Thus $E_{q}\left(\left(q^{e} \beta-\tau+1\right) \beta \frac{\partial q^{e}}{\partial z}\right)$ can be approximated to an arbitrary level of accuracy by ignoring this point. In equilibrium, $z=z^{e}$, implying that $\frac{\partial q^{e}}{\partial z}=-\frac{\tau}{\beta}$ anywhere outside the aforementioned point. Thus, the first-order condition can be solved using $\frac{\partial q^{e}}{\partial z}=-\frac{\tau}{\beta}$ and we should further analyze separately non-local deviations.

To be more specific, we can assess $E_{q}\left(\left(q^{e} \beta-\tau+1\right) \beta \frac{\partial q^{e}}{\partial z}\right)$ by treating each of the differentiable parts of $q^{e}$ separately. That is, $E_{q}\left(\left(q^{e} \beta-\tau+1\right) \beta \frac{\partial q^{e}}{\partial z}\right)=a\left(z, z^{e}\right) E_{q}\left(\left(q^{e} \beta-\tau+1\right) \beta \frac{\partial q^{e}}{\partial z} \mid q^{e}=0\right)$ $+b\left(z, z^{e}\right) E_{q}\left(\left(q^{e} \beta-\tau+1\right) \beta \frac{\partial q^{e}}{\partial z} \mid q^{e}=2\right)+c\left(z, z^{e}\right) E_{q}\left(\left(q^{e} \beta-\tau+1\right) \beta \frac{\partial q^{e}}{\partial z} \mid q^{e} \in(0,2)\right)$, where $a\left(z, z^{e}\right)$, $b\left(z, z^{e}\right)$, and $c\left(z, z^{e}\right)$ capture the probabilities that $q$ is such that we are in each of the regions. Next, we recall that, as long as $\frac{\beta q-\tau\left(z-z^{e}\right)}{\beta} \in[0,2], q^{e}(z)=\frac{\beta q-\tau\left(z-z^{e}\right)}{\beta}$ and thus $\left(q-q^{e}\right)=\frac{\tau}{\beta}\left(z-z^{e}\right)$. Because $z, z^{e} \in[0,1]$, we have that $\left|q-q^{e}\right| \in\left[0, \frac{\tau}{\beta}\right]$. As a result, either $a\left(z, z^{e}\right)=0$ or $b\left(z, z^{e}\right)=0$, and $a\left(z, z^{e}\right)+b\left(z, z^{e}\right)=\min \left\{\frac{1}{2} \frac{\tau}{\beta}\left|z-z^{e}\right|, 1\right\}$ and $c\left(z, z^{e}\right)=1-\min \left\{\frac{1}{2} \frac{\tau}{\beta}\left|z-z^{e}\right|, 1\right\}$.

Noting further that $E_{q}\left(\left(q^{e} \beta-\tau+1\right) \beta \frac{\partial q^{e}}{\partial z} \mid q^{e}=0, \frac{\beta q-\tau\left(z-z^{e}\right)}{\beta} \neq 0\right)=$ $E_{q}\left(\left(q^{e} \beta-\tau+1\right) \beta \frac{\partial q^{e}}{\partial z} \mid q^{e}=2, \frac{\beta q-\tau\left(z-z^{e}\right)}{\beta} \neq 2\right)=0$, we get that $E_{q}\left(\left(q^{e} \beta-\tau+1\right) \beta \frac{\partial q^{e}}{\partial z}\right)$ $=\left(1-\min \left\{\frac{1}{2} \frac{\tau}{\beta}\left|z-z^{e}\right|, 1\right\}\right) E_{q}\left(\left(\frac{\beta q-\tau\left(z-z^{e}\right)}{\beta} \beta-\tau+1\right) \beta\left(-\frac{\tau}{\beta}\right) \mid \frac{\beta q-\tau\left(z-z^{e}\right)}{\beta} \in(0,2), q \in[0,2]\right)$ $=-\tau\left(1-\min \left\{\frac{1}{2} \frac{\tau}{\beta}\left|z-z^{e}\right|, 1\right\}\right)\left(1-\tau-\tau\left(z-z^{e}\right)+\beta E_{q}\left(q \mid \frac{\beta q-\tau\left(z-z^{e}\right)}{\beta} \in(0,2), q \in[0,2]\right)\right)$ $=-\tau\left(1-\min \left\{\frac{1}{2} \frac{\tau}{\beta}\left|z-z^{e}\right|, 1\right\}\right)\left(1-\tau-\tau\left(z-z^{e}\right)+\beta E_{q}\left(q \mid \frac{\beta q-\tau\left(z-z^{e}\right)}{\beta} \in(0,2), q \in[0,2]\right)\right)$ $=-\tau\left(1-\min \left\{\frac{1}{2} \frac{\tau}{\beta}\left|z-z^{e}\right|, 1\right\}\right)\left(1-\tau-\tau\left(z-z^{e}\right)+\beta E_{q}\left(q \mid q \in\left(\frac{\tau\left(z-z^{e}\right)}{\beta}, 2+\frac{\tau\left(z-z^{e}\right)}{\beta}\right) \cap[0,2]\right)\right)$.

We next find the expressions for $E_{q}\left(\left(q^{e} \beta-\tau+1\right) \beta \frac{\partial q^{e}}{\partial z}\right)$ when $z=z^{e}, z>z^{e}$, and $z<z^{e}$ :

$E_{q}\left(\left(q^{e} \beta-\tau+1\right) \beta \frac{\partial q^{e}}{\partial z} \mid z=z^{e}\right)=-\tau\left(1-\tau+\beta E_{q}(q \mid q \in(0,2))\right)=-\tau(1-\tau+\beta) ;$ $E_{q}\left(\left(q^{e} \beta-\tau+1\right) \beta \frac{\partial q^{e}}{\partial z} \mid z>z^{e}\right)=-\tau\left(1-\min \left\{\frac{1}{2} \frac{\tau}{\beta}\left(z-z^{e}\right), 1\right\}\right)\left(1-\tau-\tau\left(z-z^{e}\right)\right.$ $\left.+\beta E_{q}\left(q \mid q \in\left(\frac{\tau\left(z-z^{e}\right)}{\beta}, 2\right]\right)\right)=-\tau\left(1-\min \left\{\frac{1}{2} \frac{\tau}{\beta}\left(z-z^{e}\right), 1\right\}\right)\left(1-\tau+\beta-\frac{\tau\left(z-z^{e}\right)}{2}\right)$; $E_{q}\left(\left(q^{e} \beta-\tau+1\right) \beta \frac{\partial q^{e}}{\partial z} \mid z<z^{e}\right)=-\tau\left(1-\min \left\{\frac{1}{2} \frac{\tau}{\beta}\left(z^{e}-z\right), 1\right\}\right)\left(1-\tau-\tau\left(z-z^{e}\right)\right.$ $\left.+\beta E_{q}\left(q \mid q \in\left[0,2+\frac{\tau\left(z-z^{e}\right)}{\beta}\right)\right)\right)=-\tau\left(1-\min \left\{\frac{1}{2} \frac{\tau}{\beta}\left(z^{e}-z\right), 1\right\}\right)\left(1-\tau+\beta-\frac{\tau\left(z-z^{e}\right)}{2}\right)$. 
Put differently

$$
E_{q}\left(\left(q^{e} \beta-\tau+1\right) \beta \frac{\partial q^{e}}{\partial z}\right)=-\tau\left(1-\min \left\{\frac{1}{2} \frac{\tau}{\beta}\left(z^{e}-z\right), 1\right\}\right)\left(1-\tau+\beta-\frac{\tau\left(z-z^{e}\right)}{2}\right) .
$$

We now go back to analyzing the first-order condition with respect to $z$, that is,

$$
\begin{aligned}
& p\left(\left(1+\beta-z^{e} \tau-p\right)\right)+\frac{\partial E_{q}\left(p_{2}^{A R}\left(1+\beta q^{e}-\tau-p_{2}^{A R}\right)\right)}{\partial z}=A^{\prime}(z), \text { or } \\
& p\left(\left(1+\beta-z^{e} \tau-p\right)\right)+\frac{1}{2} E_{q}\left(\left(q^{e} \beta-\tau+1\right) \beta \frac{\partial q^{e}}{\partial z}\right)=A^{\prime}(z) .
\end{aligned}
$$

Noting that in equilibrium $z=z^{e}$, and denoting the equilibrium candidate value of $z$ by $z^{A R}$. The first-order condition must satisfy

$$
p\left(1+\beta-z^{A R} \tau-p\right)-\frac{\tau(1-\tau+\beta)}{2}=A^{\prime}\left(z^{A R}\right) .
$$

Because the LHS is decreasing in $z$ and the RHS is increasing in $z$ this has a unique solution. Substituting in the first-order condition for $p_{1}^{A R}$ we get $\left(\frac{1+\beta-z^{A R} \tau}{2}\right)^{2}-\tau \frac{(1+\beta-\tau)}{2}=A^{\prime}\left(z^{A R}\right)$.

We have established that the first-order condition above is a necessary equilibrium condition. To prove that it is also a sufficient equilibrium condition, we are left to prove that the resulting $z$ survives large deviations. There is no need to worry about a deviation to a lower level of $z$ because having a range of values of $q$ for which a change in $z$ does not affect $q^{e}$ reduces the firm's incentive to decrease $z$ in order to increase $q^{e}$. This can be made apparent by noting that $E_{q}\left(\left(q^{e} \beta-\tau+1\right) \beta \frac{\partial q^{e}}{\partial z} \mid z<z^{e}\right)>E_{q}\left(\left(q^{e} \beta-\tau+1\right) \beta \frac{\partial q^{e}}{\partial z} \mid z=z^{e}\right)$.

Suppose next that the firm considers a large deviation to some $\widehat{z}>z^{A R}$. Plugging $z^{A R}$ as $z^{e}$ and $\widehat{z}$ as the decision variable, condition (11) becomes

$$
p\left(1+\beta-z^{A R} \tau-p\right)-\frac{1}{2} \tau\left(1-\min \left\{\frac{1}{2} \frac{\tau}{\beta}\left(\widehat{z}-z^{A R}\right), 1\right\}\right)\left(1-\tau+\beta-\frac{\tau\left(\widehat{z}-z^{A R}\right)}{2}\right)=A^{\prime}(\widehat{z})
$$

Note first that $\widehat{z}=z^{A R}$ provides a solution to this condition. Therefore, it is sufficient to show that the second-order condition holds. The second-order condition for the case that $\widehat{z}>z^{A R}$ is $\frac{\tau^{2}}{4 \beta}\left(1+2 \beta-\tau-\tau\left(\widehat{z}-z^{A R}\right)\right)-A^{\prime \prime}(\widehat{z})<0$ if $\frac{1}{2} \frac{\tau}{\beta}\left(\widehat{z}-z^{A R}\right)<1$, and it is $-A^{\prime \prime}(\widehat{z})<0$ if $\frac{1}{2} \frac{\tau}{\beta}\left(\widehat{z}-z^{A R}\right)>1$. The latter condition always holds because $A(\cdot)$ is convex. To see that the former holds, note that $\frac{\tau^{2}}{4 \beta}\left(1+2 \beta-\tau-\tau\left(\widehat{z}-z^{A R}\right)\right)-A^{\prime \prime}(\widehat{z})<\frac{\tau^{2}}{4 \beta}(1+2 \beta-\tau)-A^{\prime \prime}(\widehat{z})$. Then, recall 
that for all $z, \frac{\tau}{\beta}<A^{\prime \prime}(z)$ to get that

$$
\begin{aligned}
& \frac{\tau^{2}}{4 \beta}(1+2 \beta-\tau)-A^{\prime \prime}(\widehat{z})<\frac{\tau}{\beta} \frac{\tau}{4}(1+2 \beta-\tau)-\frac{\tau}{\beta}=\frac{\tau}{\beta}\left(\frac{\tau}{4}(1+2 \beta-\tau)-1\right) \\
< & \frac{\tau}{\beta}\left(\frac{0.2}{4}(1+0.4)-1\right)=\frac{\tau}{\beta}(0.07-1)<0 .
\end{aligned}
$$

Finally, we are required to show that a deviation to $\widehat{z}$ that lies exactly on the boundary condition $\frac{1}{2} \frac{\tau}{\beta}\left(\widehat{z}-z^{A R}\right)=1$ is not profitable. This is always the case because the first-order condition when $\frac{1}{2} \frac{\tau}{\beta}\left(\widehat{z}-z^{A R}\right)=1$ prescribes that $\widehat{z}<z^{A R}$.

Lemma 3 In the Reviews case, the equilibrium price and advertising strategies solve the following equations: $p_{1}^{R}=\frac{1+\beta-z^{R} \tau}{2}, p_{2}^{R}=\frac{1+\beta q-\tau}{2},\left(\frac{1+\beta-z^{R} \tau}{2}\right)^{2}=A^{\prime}\left(z^{R}\right)$. That is, $p_{1}^{R}=p_{1}^{0}<p_{1}^{A R}$ and $z^{R}=$ $z^{0}>z^{A R}$.

Proof. The derivation of $p_{2}^{R}$ is identical to the derivation of $p_{2}^{A R}$, substituting $q$ for $q^{e}$.

In period 1 the firm solves $\left(z^{R}, p_{1}^{R}\right)=\arg \max _{z, p} \Pi=p D_{1}(z, p)+E_{q}\left(p_{2}^{R} D_{2}^{R}\left(p_{2}^{R}\right)\right)-A(z)$ or $\left(z^{R}, p_{1}^{R}\right)=\arg \max _{z, p} \Pi=p\left(z\left(1+\beta-z^{e} \tau-p\right)\right)+E_{q}\left(p_{2}^{R}\left(1+\beta q-\tau-p_{2}^{R}\right)\right)-A(z)$. We note that $E_{q}\left(p_{2}^{R}\left(1+\beta q-\tau-p_{2}^{R}\right)\right)$ is independent of $(z, p)$ and therefore $p_{1}^{R}$ and $z^{R}$ are chosen to maximize period 1 profits and we get $p_{1}^{R}=\frac{1+\beta-z^{R} \tau}{2}$ and $p_{1}^{R}\left(1+\beta-z^{R} \tau-p_{1}^{R}\right)=A^{\prime}\left(z^{R}\right)$. Substituting in the first-order condition for $p_{1}^{R}$ we get $\left(\frac{1+\beta-z^{R} \tau}{2}\right)^{2}=A^{\prime}\left(z^{R}\right)$. Thus, $z^{R}=z^{0}$ and $p_{1}^{R}=p_{1}^{0}$ with the implied relationships to $z^{A R}$ and $p_{1}^{A R}$.

Proof of Proposition 2. The proof follows from three lemmas (Lemmas 4-6).

Lemma 4 In the Benchmark case, $\mu_{2}^{0}<\mu_{1}^{0}$.

Proof. We show that $\mu_{2}^{0}<\mu_{1}\left(z^{0}\right)$. Recall that $p_{2}^{0}=p_{1}^{0}-\frac{\left(1-z^{0}\right) \tau}{2}$. Thus, denoting $p_{1}^{0}=p$ and $z^{0}=z$, the condition $\mu_{2}^{0}<\mu_{1}\left(z^{0}\right)$ becomes $\frac{1-\left(p-\frac{(1-z) \tau}{2}\right)-\tau+(2 q-1) \beta}{2}<\frac{1+\beta(2 q-1)-z \tau-p}{2}$ or $1>z$ which always holds.

Lemma 5 Let $\bar{q}^{A R}=1+\frac{\tau}{3 \beta}\left(z^{A R}-1\right)$ and note that $\bar{q}^{A R}<1$ for all $z^{A R} \in(0,1)$. In the AR case, $\mu_{2}^{A R}<\mu_{1}^{A R}$ if and only if $q>\bar{q}^{A R}$.

Proof. In equilibrium, the condition for $\mu_{2}^{A R}<\mu_{1}^{A R}$ is $\frac{1+\beta q-\tau-p_{2}}{2}<\frac{1+\beta(2 q-1)-z \tau-p_{1}}{2}$. Noting that $p_{2}^{A R}=p_{1}^{A R}+\frac{\beta(q-1)-\tau(1-z)}{2}$ the condition becomes $\frac{1+\beta q-\tau-\left(p_{1}+\frac{\beta(q-1)-\tau(1-z)}{2}\right)}{2}<\frac{1+\beta(2 q-1)-z \tau-p_{1}}{2}$ or $1+\frac{\tau}{3 \beta}(z-1)<q$. 
Lemma 6 In the Reviews case, there exists $\bar{q}^{R}$ such that $\mu_{2}^{R}<\mu_{1}^{R}$ if and only if $q>\bar{q}^{R}$. Moreover, $\bar{q}^{R} \geq \bar{q}^{A R}$. That is, ratings increase for a larger range of qualities than in the AR case.

Proof. The mean utility of a second-generation purchasing consumers is

$$
\mu_{2}^{R}=\frac{3\left(p_{2}^{R}\right)^{2}-6 p_{2}^{R} q \beta+6 p_{2}^{R} \tau-6 p_{2}^{R}+3 q^{2} \beta^{2}-6 q \beta \tau+6 q \beta+4 \tau^{2}-6 \tau+3}{6\left(1+\beta q-p_{2}^{R}-\tau\right)} .
$$

Recall that $p_{2}^{A R}=p_{2}^{R}$ and set $p=p_{2}^{A R}=p_{2}^{R}$, we get:

$$
\mu_{2}^{R}=\frac{3 p^{2}-6 p q \beta+6 p \tau-6 p+3 q^{2} \beta^{2}-6 q \beta \tau+6 q \beta+4 \tau^{2}-6 \tau+3}{6(1+\beta q-p-\tau)}>\frac{1+\beta q-\tau-p}{2}=\mu_{2}^{A R} .
$$

Note that with some algebra the above inequality becomes $\frac{1}{3} \tau^{2}>0$, which always holds. In addition, we have $\mu_{1}^{A R} \geq \mu_{1}^{R}$. To see why recall that $z^{A R}<z^{R}$ and note that in both the $\mathrm{AR}$ and review treatments $p_{1}=\frac{1+\beta-z \tau}{2}$ and therefore, $\mu_{1}(z)=\frac{1+\beta(2 q-1)-z \tau-p_{1}}{2}=\frac{1+\beta(2 q-1)-z \tau-\frac{1+\beta-z \tau}{2}}{2}$

$=q \beta-\frac{3}{4} \beta-\frac{1}{4} z \tau+\frac{1}{4}$ which is decreasing in $z$. Subsequently, for any $q$ such that $\mu_{1}^{A R}<\mu_{2}^{A R}$ it is also true that $\mu_{1}^{R}<\mu_{2}^{R}$, and for any $q$ for which $\mu_{1}^{R}>\mu_{2}^{R}$ it is also true that $\mu_{1}^{A R}>\mu_{2}^{A R}$. That is, if there exists $\bar{q}^{R}$ such that in the Reviews case the average rating of second-generation buyers is lower than the average rating of first-generation buyers if and only if $q>\bar{q}^{R}$, then it must be the case that $\bar{q}^{R} \geq \bar{q}^{A R}$.

To show that there exists such a $\bar{q}^{R}$ we show that $\mu_{2}^{R}-\mu_{1}(z)$ is monotonically decreasing in $q$ for any $z$. To do that, note that for a general $q, p_{2}^{R}=p_{1}^{R}+\frac{q \beta-\beta+z \tau-\tau}{2}$. We next prove that $\mu_{2}^{R}-\mu_{1}(z)$ is monotonically decreasing in $q$ for any $z$ in two steps. First we prove the hypothetical claim that if $p_{2}^{R}=p_{1}^{R}$ then $\mu_{2}^{R}-\mu_{1}(z)$ is monotonically decreasing in $q$ for any $z$. Then, we show that, holding fixed $p_{1}^{R}, \mu_{2}^{R}$ is monotonically decreasing in $p_{2}^{R}-p_{1}^{R}$ for any $z$ (note that $p_{2}^{R}-p_{1}^{R}$ is increasing in $q$ ). Given that $\mu_{1}(z)$ is independent of $p_{2}^{R}-p_{1}^{R}$ conditional on $p_{1}^{R}$, this completes the proof as required.

We begin by showing that $\frac{\partial\left(\left(\mu_{2}^{R}-\mu_{1}(z)\right) \mid p_{2}^{R}=p_{1}^{R}=p\right)}{\partial q}=-\frac{1}{6} \frac{\beta\left(3 p^{2}-6 p q \beta+6 p \tau-6 p+3 q^{2} \beta^{2}-6 q \beta \tau+6 q \beta+4 \tau^{2}-6 \tau+3\right)}{(p+\tau-q \beta-1)^{2}}$ and has the opposite sign of $\left(3 p^{2}-6 p q \beta+6 p \tau-6 p+3 q^{2} \beta^{2}-6 q \beta \tau+6 q \beta+4 \tau^{2}-6 \tau+3\right)$, which, in turn, is increasing in $q$. Therefore, it is sufficient to show that $\left(3 p^{2}-6 p q \beta+6 p \tau-6 p+3 q^{2} \beta^{2}-\right.$ $\left.6 q \beta \tau+6 q \beta+4 \tau^{2}-6 \tau+3>0\right)$ for $q=0$. That is, to show that $\left(3 p^{2}+6 p \tau-6 p+4 \tau^{2}-6 \tau+3>0\right)$, which holds because $\left(3 p^{2}+6 p \tau-6 p+4 \tau^{2}-6 \tau+3\right)$ is decreasing in $p$, and when substituting $p=1$ we get $4 \tau^{2}>0$, which always holds.

Finally we show that, holding fixed $p_{1}^{R}, \mu_{2}^{R}$ is monotonically decreasing in $p_{2}^{R}-p_{1}^{R}$ for any $z$, which is equivalent to showing that $\mu_{2}^{R}$ decreases in $p_{2}^{R}$, which, in turn, holds by construction. 
Proof of Proposition 3. We first prove that $\Pi^{R}>\Pi^{0}$ using a revealed preference argument. We note that if the firm were to choose in the Reviews case the same period 2 price as in the Benchmark case, the profits would have been identical in the Reviews and Benchmark cases. We then note that in the Reviews case the firm is able to choose the same price as in the Benchmark regardless of the quality, whereas the reverse is not true, in the Benchmark case the firm is not able to replicate the period 2 pricing of the Reviews case. The concavity of the profit function in the second period price suggests that the firm is choosing the unique profit maximizing second period price in each case. Thus, $\Pi^{R}>\Pi^{0}$ as required.

To prove the claim for the relationship between $\Pi^{R}$ and $\Pi^{A R}$, note that in equilibrium, second period revenues are always the same in the Reviews and AR cases. Subsequently, in equilibrium, $\Pi^{R}>\Pi^{A R}$ if and only if first period profit is higher in the Reviews case. That is, if $p_{1}^{R}\left(z^{R}\left(1+\beta-z^{R} \tau-p_{1}^{R}\right)\right)-A\left(z^{R}\right)>p_{1}^{A R}\left(z^{A R}\left(1+\beta-z^{A R} \tau-p_{1}^{A R}\right)\right)-A\left(z^{A R}\right)$ or, substituting in $p_{1}^{R}$ and $p_{1}^{A R}$ and rearranging $z^{R}\left(\frac{1+\beta-z^{R} \tau}{2}\right)^{2}-A\left(z^{R}\right)>z^{A R}\left(\frac{1+\beta-z^{A R} \tau}{2}\right)^{2}-A\left(z^{A R}\right)$. Let $z^{*}=\arg \max _{z} z\left(\frac{1+\beta-z \tau}{2}\right)^{2}-A(z)$. Then $z^{*}$ solves $\left(\frac{1+\beta-z^{*} \tau}{2}\right)^{2}-\tau z^{*}\left(\frac{1+\beta-z^{*} \tau}{2}\right)=A^{\prime}\left(z^{*}\right)$, which can be re-written as follows

$$
\left(\frac{1+\beta-z^{*} \tau}{2}\right)^{2}-\tau\left(\frac{1+\beta-z^{*} \tau}{2}\right)+\tau\left(1-z^{*}\right)\left(\frac{1+\beta-z^{*} \tau}{2}\right)=A^{\prime}\left(z^{*}\right)
$$

Recall that $z^{R}$ and $z^{A R}$ satisfy $\left(\frac{1+\beta-z^{R} \tau}{2}\right)^{2}=A^{\prime}\left(z^{R}\right)$ and $\left(\frac{1+\beta-z^{A R} \tau}{2}\right)^{2}-\tau \frac{(1+\beta-\tau)}{2}=A^{\prime}\left(z^{A R}\right)$, respectively. Therefore, $z^{R}>z^{*}>z^{A R}$. Furthermore, if $z^{*} \rightarrow 1$ then the conditions for $z^{*}$ and $z^{A R}$ converge and thus $z^{*}=z^{A R}$. And similarly, if $z^{*} \rightarrow 0$ then the conditions for $z^{*}$ and $z^{R}$ converge and we have that $z^{*}=z^{R}$. As a result, there exist $\underline{z}, \bar{z} \in(0,1)$ such that if $z^{*}<\underline{z}$ then $\Pi^{R}>\Pi^{A R}$ and if $z^{*}>\bar{z}$ then $\Pi^{R}<\Pi^{A R}$.

Next, consider again the expression $\left(\frac{1+\beta-z^{*} \tau}{2}\right)^{2}-\tau z^{*}\left(\frac{1+\beta-z^{*} \tau}{2}\right)=A^{\prime}\left(z^{*}\right)$. The LHS decreases in $z^{*}$ and the RHS increases in $z^{*}$. Therefore, any parameter change that increases the LHS leads to an increase in $z^{*}$ and any change that increases the RHS leads to a decrease in $z^{*}$. This explains the effect of the advertising cost function on when $z^{*} \rightarrow 1$ vs. $z^{*} \rightarrow 0$.

A simple derivative shows that the LHS decreases in $\tau$ and increases in $\beta$. Therefore, $z^{*}$ decreases in $\tau$ and increases in $\beta$. Subsequently,

1. for any $A^{\prime}$ and $\beta$, there exists $[\underline{\tau}, \bar{\tau}]$ such that if $\tau<\underline{\tau}$ then $z^{*}$ is sufficiently large so that $\Pi^{A R}>\Pi^{R}$ and if $\tau>\bar{\tau}$ then $z^{*}$ is sufficiently small so that $\Pi^{R}>\Pi^{A R}$. 
2. for any $A^{\prime}$ and $\tau$, there exists $\left[\beta, \bar{\beta}\right.$ such that if $\beta<\underline{\beta}$ then $z^{*}$ is sufficiently small so that $\Pi^{R}>\Pi^{A R}$ and if $\beta>\bar{\beta}$ then $z^{*}$ is sufficiently large so that $\Pi^{A R}>\Pi^{R}$.

Finally, we prove that $E\left(C S^{R}\right)>E\left(C S^{0}\right)$ and $E\left(C S^{R}\right)>E\left(C S^{A R}\right)$. To see that $E\left(C S^{R}\right)>$ $E\left(C S^{0}\right)$, note first that the identical period 1 price and advertising interval implies that the first period consumer surplus is the same in the Benchmark and Reviews cases. To prove that the expected period 2 consumer surplus is higher in the Review case, it is sufficient to show that: (1) $\left.\mu_{2}^{R}\right|_{q=1}>\left.\mu_{2}^{0}\right|_{q=1}$, and $(2) \mu_{2}^{R}$ is increasing and convex in $q$. This is sufficient because we know that $E_{q}\left(D_{2}^{R}\right)=D_{2}^{0}$ and $D_{2}^{R}$ is increasing in $q$.

(1) $\left.\mu_{2}^{R}\right|_{q=1}>\left.\mu_{2}^{0}\right|_{q=1}:\left.\mu_{2}^{0}\right|_{q=1}=\frac{1-\tau+\beta}{4}$ and $\left.\mu_{2}^{R}\right|_{q=1}=\frac{3 \beta^{2}-6 \beta \tau+6 \beta+7 \tau^{2}-6 \tau+3}{12(1-\tau+\beta)}$, therefore $\left.\mu_{2}^{R}\right|_{q=1}-$ $\left.\mu_{2}^{0}\right|_{q=1}=\frac{1}{3} \frac{\tau^{2}}{\beta-\tau+1}>0$ as required.

(2) $\mu_{2}^{R}$ is increasing and convex in $q$ : Substituting $p_{2}^{R}=\frac{1+\beta q-\tau}{2}$ into $\mu_{2}^{R}=\frac{3\left(p_{2}^{R}\right)^{2}-6 p_{2}^{R} q \beta+6 p_{2}^{R} \tau-6 p_{2}^{R}+3 q^{2} \beta^{2}-6 q \beta \tau+6 q \beta+4 \tau^{2}-6 \tau+3}{6\left(1+\beta q-p_{2}^{R}-\tau\right)}$ and taking the derivative with respect to $q$ we get $\frac{d \mu_{2}^{R}}{d q}=\frac{\beta}{12} \frac{\left(3 q^{2} \beta^{2}-6 q \beta \tau+6 q \beta-\tau^{2}-6 \tau+3\right)}{(q \beta-\tau+1)^{2}}>0$, where the inequality holds because $\frac{d \mu_{2}^{R}}{d q}$ has the same sign as $3 q^{2} \beta^{2}-6 q \beta \tau+6 q \beta-\tau^{2}-6 \tau+3=3+3 q^{2} \beta^{2}-\tau^{2}-6 \tau+6 q \beta(1-\tau)>3-\tau^{2}-6 \tau$ and $\tau<\frac{1}{5}$. Furthermore, $\frac{d^{2} \mu_{2}^{R}}{d q^{2}}=\frac{2}{3} \beta^{2} \frac{\tau^{2}}{(q \beta-\tau+1)^{3}}>0$.

To see that $E\left(C S^{R}\right)>E\left(C S^{A R}\right)$, we note that in period 1 the AR case exhibits a higher price and lower advertising interval. In period 2 the AR and Reviews cases have the same price and the same number of purchases, but in the Reviews case demand is more centered around the correct location and therefore generates higher consumers utilities on average, as captured by the higher average ratings (see proof of Lemma 6). 


\section{References}

Barnes, Brooks (2011), ' 'Green Lantern' fizzles at the box office', The New York Times . June, 19.

Bass, Frank M., Dipak C. Jain and Trichy Krishnan (1994), 'Why the bass model fits without decision variables', Marketing Science 13(3), 203-223.

Brandes, Leif, David Godes and Dina Mayzlin (2020), 'What drives extremity bias in online reviews? theory and experimental evidence', Working Paper.

CarGurus (2006), '2001 Ford Escape overview', Car Gurus . November, 2.

Chen, Yubo and Jinhong Xie (2008), 'Online consumer review: Word-of-mouth as a new element of marketing communication mix', Management Science 54(3), 477-491.

Chevalier, Judith A. and Dina Mayzlin (2006), 'The effect of word of mouth on sales: Online book reviews', Journal of Marketing Research 43(3), 345-354.

Chintagunta, Pradeep K., Shyam Gopinath and Sriram Venkataraman (2010), 'The effects of online user reviews on movie box office performance: Accounting for sequential rollout and aggregation across local markets', Marketing Science 29(5), 944-957.

Del Rey, Jason (2020), 'Amazon can't end fake reviews, but its new system might drown them out', Vox . February, 14.

Godes, David B. (2017), 'Product policy in markets with word-of-mouth communication', Management Science 63(1), 267-278.

Godes, David B. and Jose C. Silva (2012), 'Sequential and temporal dynamics of online opinion', Marketing Science 31(3), 448-473.

Hughes, Mark (2017), ' 'Wonder Woman’ powering to $\$ 800$ million this weekend', Forbes . August, 18.

Jiang, Baojun and Bicheng Yang (2019), 'Quality and pricing in a market with information sharing', Management Science 65(1), 272-285.

Joshi, Yogesh and Andres Musalem (2020), 'When consumers learn, money burns: Signaling quality via advertising with observational learning and word of mouth', Marketing Science. Forthcoming.

Kuksov, Dmitri and Chenxi Liao (2019), 'Opinion leaders and product variety', Marketing Science 38(5), 812-834. 
Kuksov, Dmitri and Ying Xie (2010), 'Pricing, frills, and customer ratings', Marketing Science 29(5), 925-943.

Mankiw, Gregory N. (1985), 'Small menu costs and large business cycles: A macroeconomic model of monopoly', The Quarterly Journal of Economics 100(2), 529-538.

Moon, Sangkil, Yoonseo Park and Yong Seog Kim (2014), 'The impact of text product reviews on sales', European Journal of Marketing 48(11/12), 2176-2197.

Nair, Harikesh (2019), Diffusion and Pricing Over the Product Life Cycle, Elsevier, chapter Handbook of the Economics of Marketing.

Rocklage, Matthew D. and Russell H. Fazio (2020), 'The enhancing versus backfiring effects of positive emotion in consumer reviews', Journal of Marketing Research 57(2), 332-352.

Schoenmuller, Verena, Oded Netzer and Florian Stahl (2020), 'The polarity of online reviews: Prevalence, drivers and implications', Journal of Marketing Research . Forthcoming.

Sun, Monic (2012), 'How does the variance of product ratings matter?', Management Science 58(4), 696-707.

Taylor, John B. (1980), 'Aggregate dynamics and staggered contracts', Journal of Political Economy 88(1), 1-23.

Ulrich, Karl and Steven Eppinger (2015), Product Design and Development, sixth edn, McGraw-Hill Education.

Urban, Glenn L. and John R. Hauser (1993), Design and Marketing of New Products, second edn, Prentice-Hall.

Warren, Tom (2017), 'Microsoft confirms Surface Book 2's power problem', The Verge . November, 21.

Wiggers, Kyle (2017), 'Everything we know about Samsung's Galaxy Note 7 recall', Digital Trends . May, 17. 